\title{
Seismic vulnerability of circular tunnels in sand
}

\author{
FILOMENA DE SILVA*, STEFANIA FABOZZI†, NIKOLAOS NIKITAS \\ EMILIO BILOTTA§ and RAUL FUENTES $\|$
}

\begin{abstract}
The paper is focused on the assessment of seismic fragility curves for circular tunnels under moderate to severe earthquakes with the aim of improving the reliability of the risk assessment of underground infrastructural networks. Non-linear two-dimensional dynamic analyses were performed on different tunnel and soil configurations by using the finite-difference method implemented in Flac2D software. The applied input motions were selected considering their amplitude and frequency content variability. The response accelerations and predominant frequencies computed at ground level, above the tunnel, were compared with the corresponding free-field results to distinguish the effects attributable to the tunnel presence from those due to the site amplification. Tunnel safety was assessed through fragility curves, taking into account the dependence of tunnel lining bending resistance on the axial force variation during the earthquake. The more effective intensity measure was investigated correlating the tunnel performance to peak ground accelerations and peak ground velocities computed at the ground level and at the bedrock depth, respectively. The resulting fragility curves showed a satisfying matching with the empirical ones, generated on the basis of the observed seismic damage on tunnels.
\end{abstract}

KEYWORDS: dynamics; earthquakes; limit state design/analysis; numerical modelling; tunnels \& tunnelling

\begin{abstract}
INTRODUCTION
Nowadays, many countries in the world are facing a continuous demand for urbanisation, structuring the underground space to develop their physical interconnectivity (Broere, 2016). Lifeline networks, in particular, including transportation infrastructures and utility systems, have a strategic impact on the socio-economic conditions of a country, hence their seismic risk prediction, prevention and mitigation in the perspective of seismic urban planning and resilience is a crucial issue in seismically hazardous areas. Owing to their spatial extent and variability, linear infrastructure systems are vulnerable to multiple geotechnical hazards (i.e. seismic ground shaking, liquefaction, landslides and fault ruptures). Among other elements of such systems, tunnels of urban and extra-urban road, railway and highway transportation systems, or for other utility networks (i.e. waste water network), are particularly exposed to such hazards.

The object of this work is to develop numerical fragility curves for circular bored tunnels subjected to seismic ground shaking. This can induce damage in the tunnel structure, as observed after recent and historic seismic events (e.g. Dowding \& Rozen, 1978; Sharma \& Judd, 1991; Power et al., 1998; O'Rourke \& Liu, 1999; Corigliano, 2007). The
\end{abstract}

Manuscript received 15 February 2019; revised manuscript accepted 30 January 2020. Published online ahead of print 3 March 2020.

Discussion on this paper closes on 1 March 2022, for further details see p. ii.

Published with permission by the ICE under the CC-BY 4.0 license. (http://creativecommons.org/licenses/by/4.0/)

* University of Naples Federico II, Naples, Italy

(Orcid:0000-0003-3509-4281).

$\dagger$ National Research Council, Rome, Italy

(Orcid:0000-0002-8024-7913).

\$ University of Leeds, Leeds, UK (Orcid:0000-0002-6243-052X).

$\S$ University of Naples Federico II, Naples, Italy

(Orcid:0000-0002-3185-2738).

|| University of Leeds, Leeds, UK; Escuela Técnica Superior de Ingeniería de Caminos, Canales y Puertos, Universitat Politècnica de València, Spain (Orcid:0000-0001-8617-7381). earthquake effect on the tunnel lining is also shown by experimental evidence from centrifuge models of tunnels undergoing shaking in the transverse section (e.g. Cilingir \& Madabhushi, 2011a, 2011b; Lanzano et al., 2012), indicating that kinematic soil-structure interaction produces an increase of internal forces in the tunnel lining under dynamic shaking. Therefore, a probabilistic seismic vulnerability assessment study has been conducted for some ideal tunnel configurations, based on a numerical approach for the fragility analysis. In the perspective of the seismic risk analysis according to the principles of performance-based earthquake engineering (PBEE), the fragility function expresses the probability of exceeding different damage states (DS) for a given level of earthquake intensity measure (IM), accounting for the various uncertainties associated with the seismic hazard and the structural response estimation (Pitilakis et al., 2014).

In addition to a few empirical fragility curves (ALA, 2001; NIBS, 2004), a limited number of numerical fragility curves for circular bored tunnels are currently available in the literature, generally derived for different ground conditions and tunnel linings (Salmon et al., 2003; Argyroudis \& Pitilakis, 2012; Andreotti \& Lai, 2014, Kiani et al., 2016; Argyroudis et al., 2017; Fabozzi et al., 2017).

In this work, the cloud method (Jalayer et al., 2015) has been adopted to generate the proposed fragility curves expressed in terms of peak ground acceleration (PGA) and velocity. The method considered requires fewer seismic analyses to be performed and the use of unscaled records to avoid unrealistic and undesired modification of the scaled signals. Finally, the tunnel structure fragility was expressed in terms of the ratio between the loading and resistance bending moment, assuming its dependence on the axial force variation during earthquake.

\section{NUMERICAL MODELS}

Most of the available experimental measurements of seismic increments of the tunnel lining internal forces were 
collected in centrifuge tests on circular tunnels. Mainly for experimental reasons, dry sand was generally used in the tests to model the ground surrounding the tunnel (e.g. Cilingir \& Madabhushi, 2011a, 2011b; Lanzano et al., 2012). Such tests refer to shallow circular tunnel models (i.e. cover-to-diameter ratio, $C / D$, lower than 3 ) embedded in either dense $\left(D_{\mathrm{r}}=75 \%\right)$ or loose sand $\left(D_{\mathrm{r}}=40 \%\right)$. Although limited, the range of cases covered by such experimental evidence is large enough to represent several cases of real shallow tunnels, where the pore pressure build-up during shaking may be neglected. Consequently, for the purposes of this study, the seismic soil-tunnel interaction was evaluated with reference to geometrical schemes and ground conditions similar to the experimental cases available in the literature. Therefore, a $10 \mathrm{~m}$ outer diameter and $0.50 \mathrm{~m}$ thick circular cross-section embedded in a $60 \mathrm{~m}$ thick dry sand layer, placed on a soft rock bedrock was considered. It is worth noting that, although the effect of the construction stages on the seismic behaviour of a tunnel lining in sand has been evidenced in published numerical studies (Fabozzi \& Bilotta, 2016; Fabozzi, 2017; Bilotta, 2018), in this work the tunnel was assumed as wished in place and the excavation process was not simulated in the analyses. Two geometrical layouts were investigated, varying the tunnel axis depth from $15 \mathrm{~m}(C / D=1)$ to $30 \mathrm{~m}(C / D=2 \cdot 5)$, as shown in Fig. 1(a). The analyses were performed on two idealised soil models representative of loose and dense sand, characterised by a relative density $D_{\mathrm{r}}=40 \%$ and $D_{\mathrm{r}}=75 \%$, respectively. The assumed $D_{\mathrm{r}}$ values correspond to the void ratios, $e$, reported in Table 1 and calculated on the basis of the typical values of $e_{\min }$ and $e_{\max }$ for clean sand - that is 0.5 and 1 , respectively (Cubrinovski \& Ishihara, 2002). The dependence of the shear wave velocity, $V_{\mathrm{S}}$, on the void ratio and depth was defined through the empirical power law correlation adopted by Hardin (1978), assuming the coefficients proposed by Silvestri \& Lanzo (1999). The resulting $V_{\mathrm{S}}$ profiles are shown in Fig. 1(b), highlighting the correction (Tropeano et al., 2018) close to the ground surface to avoid the unrealistic convergence to zero (dotted lines). In more detail, a linear trend was assumed, anchored to the tangent to the $V_{\mathrm{S}}$ value at a depth of $10 \mathrm{~m}$. The depth-dependent small-strain shear stiffness, $G_{0}$, and bulk modulus, $K_{0}$, were computed from the $V_{\mathrm{S}}$ profile, setting the constant soil density, $\rho$, and Poisson's ratio, $v$, as reported in Table 1 . The shear resistance of the cohesionless loose and dense sand was assumed to be governed by the friction angle $\phi=30^{\circ}$ and $\phi=35^{\circ}$, respectively. The non-linear and dissipative soil response was inferred from the variation of the normalised shear modulus, $G / G_{0}$, and damping ratio, $D$, with shear strain, $\gamma$, resulting from laboratory tests performed on loose to dense sand by Seed \& Idriss (1970). Fig. 1(c) shows, for instance, the mean and the envelope curves of the experimental data.

A linear behaviour was assumed for the underlying soft rock, with the typical physical and mechanical properties of a seismic bedrock.

The concrete strength class C25/30 was adopted to model the tunnel lining characterised by the Young's modulus, $E$, the Poisson's ratio, $v$, the uniaxial compression, $\sigma_{\mathrm{cy}}$, and tensile strength, $\sigma_{\mathrm{t}}$, reported in Table 2 .

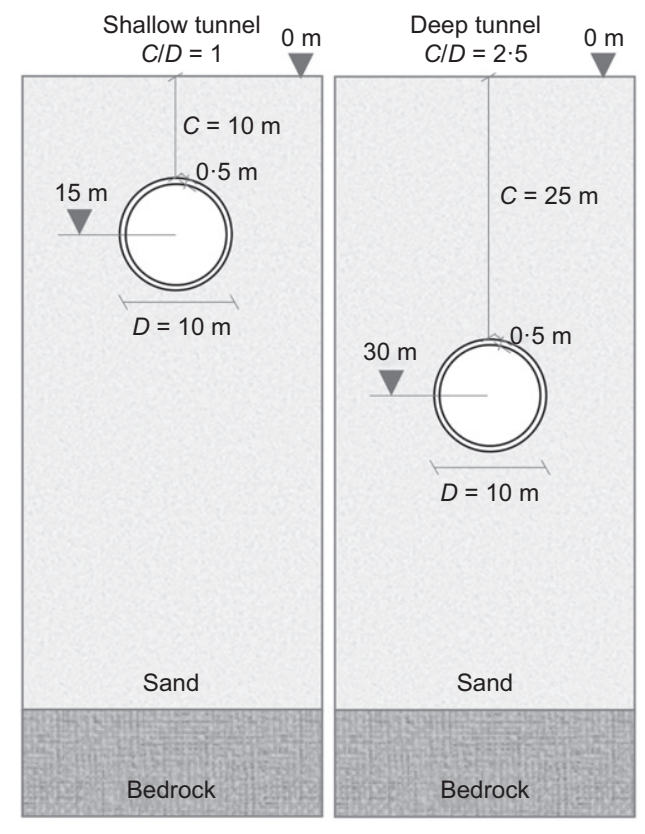

(a)

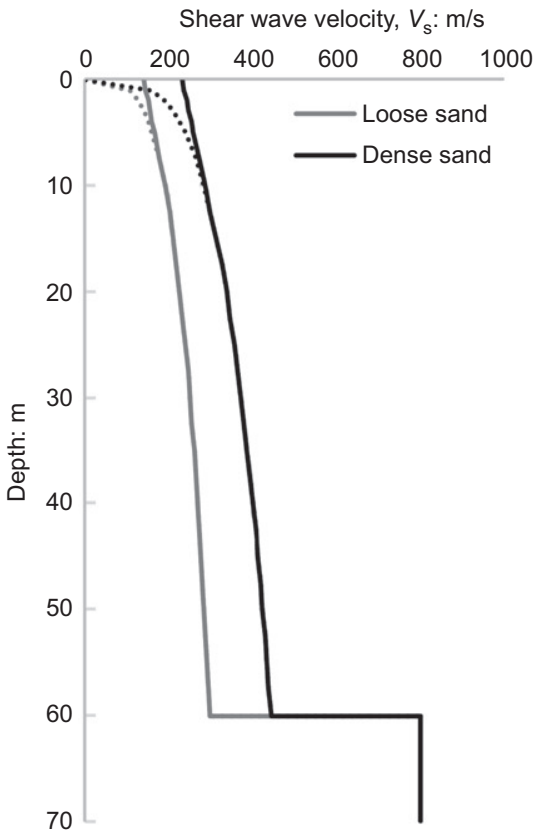

(b)

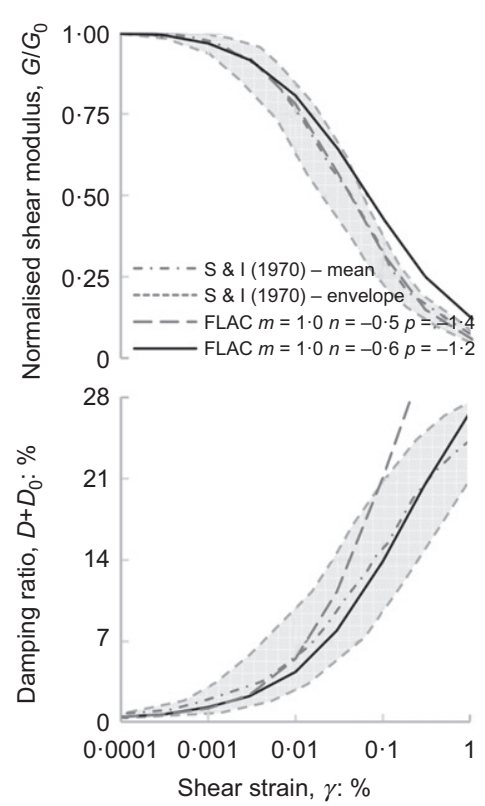

(c)

Fig. 1. (a) Geometry of the reference schemes; (b) shear wave velocity profiles of loose and dense sand; (c) variation of the normalised shear modulus and damping ratio with shear strain (note: S \& I, Seed \& Idriss)

Table 1. Physical and mechanical soil properties

\begin{tabular}{|c|c|c|c|c|c|c|c|c|}
\hline & $\rho: \mathrm{kg} / \mathrm{m}^{3}$ & $D_{\mathrm{r}}: \%$ & $e$ & $V_{\mathrm{S}}: \mathrm{m} / \mathrm{s}$ & $G_{0}: \mathrm{MPa}$ & $v$ & $K_{0}: \mathrm{MPa}$ & $\phi:$ degrees \\
\hline Loose sand & 1600 & 40 & $0 \cdot 80$ & $150-300$ & $36-144$ & $0 \cdot 33$ & $94-376$ & 30 \\
\hline Dense sand & 1800 & 75 & 0.63 & $230-450$ & 95-365 & $0 \cdot 30$ & 206-790 & 35 \\
\hline Bedrock & 2000 & - & - & 800 & 1024 & $0 \cdot 15$ & 1122 & - \\
\hline
\end{tabular}


Table 2. Mechanical properties of concrete

\begin{tabular}{l|c|c|c|c|c}
\hline$\rho: \mathrm{kg} / \mathrm{m}^{3}$ & $E: \mathrm{MPa}$ & $v$ & $D_{0}: \%$ & $\sigma_{\mathrm{cy}}: \mathrm{MPa}$ & $\sigma_{\mathrm{t}}: \mathrm{MPa}$ \\
\hline 2500 & 30000 & $0 \cdot 15$ & 5 & 25 & $2 \cdot 5$ \\
\hline
\end{tabular}

Summarising, the different tunnel depths coupled with the considered soil models led to four reference schemes

(a) shallow tunnel in loose sand

(b) shallow tunnel in dense sand

(c) deep tunnel in loose sand

(d) deep tunnel in dense sand.

Note that, even if the considered value of $C / D=2 \cdot 5$ does not correspond exactly to a deep tunnel, in the present work this definition is assumed only to distinguish the studied cases.

The two-dimensional models reproducing the four reference schemes were generated through the finite-difference code Flac (Itasca, 2011). The soil domain is $140 \mathrm{~m}$ wide and $60 \mathrm{~m}$ deep, based on the top of a stiffer $10 \mathrm{~m}$ thick layer simulating the bedrock. Solid elements were used to model the tunnel lining located in the middle of the numerical model, with the axis depth equal to $15 \mathrm{~m}$ or $30 \mathrm{~m}$ according to the geometric layout considered (see Fig. 1(a)). A no-slip condition modelled the surface contact between the lining and the soil, which seemed to be appropriate for a concrete lining cast in situ. This contact condition affects the axial loading of the tunnel lining during ground shaking, and consequently its bending capacity.

The mesh size, $\Delta$, was calibrated according to the rule $\Delta<V_{\mathrm{S}} / 8 f_{\text {max }}$ proposed by Kuhlemeyer \& Lysmer (1973), to allow the typical seismic wave frequencies up to $f_{\max }=25 \mathrm{~Hz}$ to propagate reliably through the layered soil. Smaller mesh elements, instead, were used to model the tunnel lining. The infinite extension in depth of the bedrock was simulated by means of dashpots, providing viscous normal and shear stresses proportional to the volume and shear wave velocities of the bottom layer. To minimise the numerical model size, the so-called 'free-field' boundary conditions were applied to the lateral sides, simulating an ideal horizontal soil profile connected to the main-grid domain through viscous dashpots. As an example, Fig. 2 shows the numerical model of the shallow tunnel in dense sand reference scheme.

As numerous non-linear dynamic analyses need to be performed to generate fragility curves, the constitutive model directly included in the Flac library was chosen, to limit the computational effort. The bedrock was assumed elastic, while the elastic-perfectly plastic Mohr-Coulomb

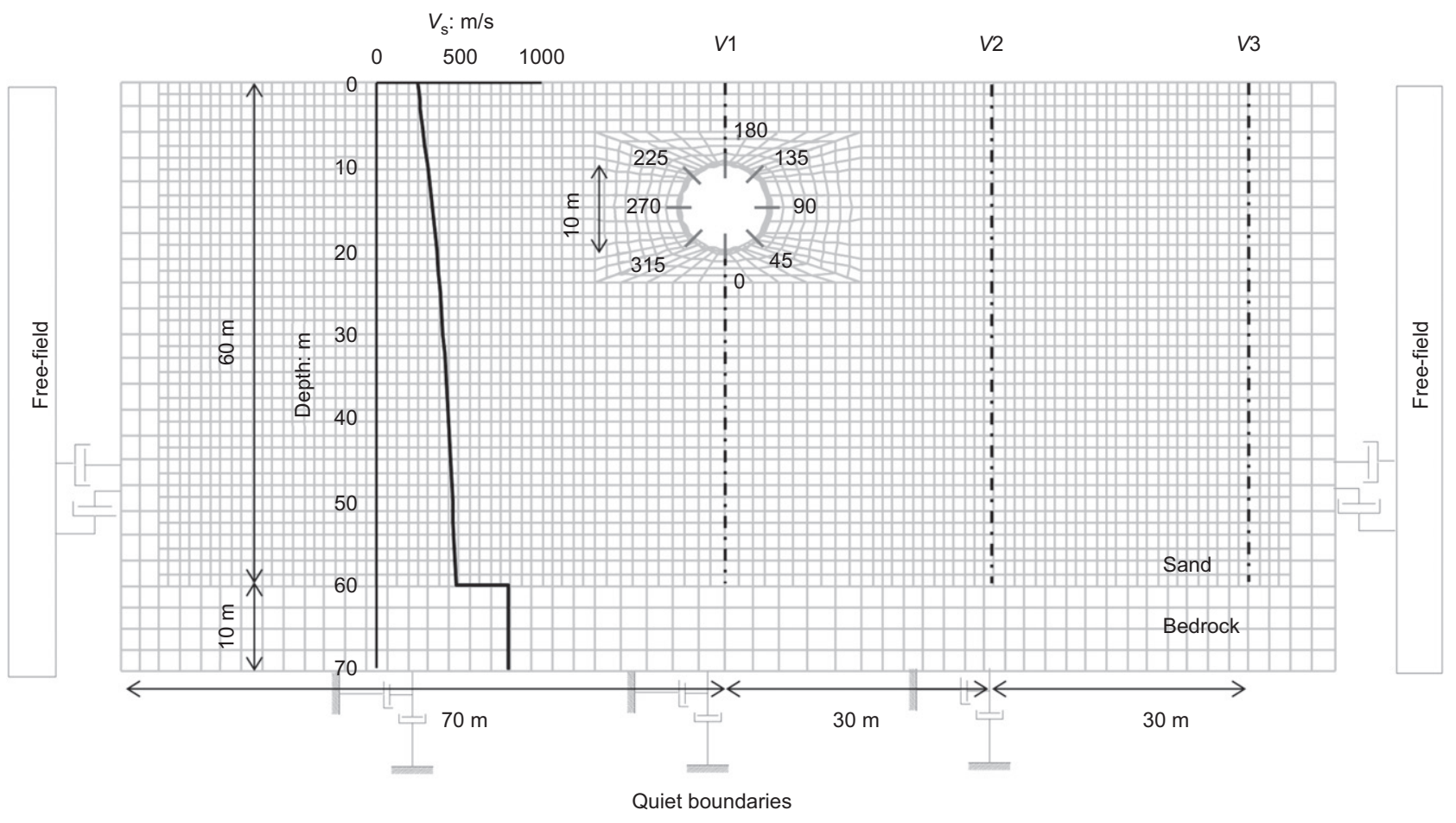

(a)

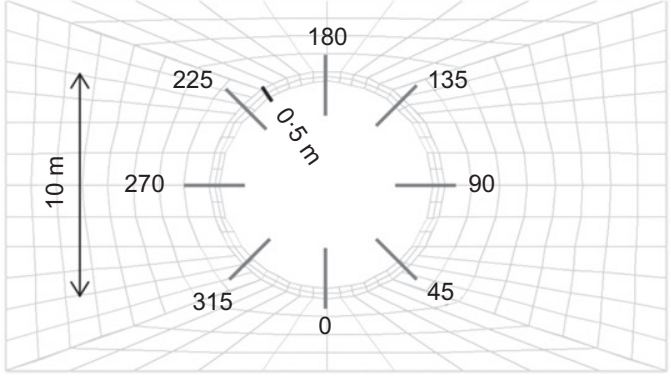

(b)

Fig. 2. (a) Numerical model of shallow tunnel in dense sand; (b) detail of the mesh around the tunnel. Control vertical sections and reference tunnel sections are superimposed on the scheme 
constitutive model with shear stiffness increasing with depth was adopted to model the sand behaviour. According to this model, yielding occurs when the maximum shear strength is attained, the latter being a function of the mean stress acting in each element of the soil model.

Conti et al. (2014) proved that, at least for the limited number of experimental cases in dry sand that were analysed, the Mohr-Coulomb model with non-linear and hysteretic behaviour provided cyclic changes of bending moment similar to those predicted by using a more refined constitutive model defined within the framework of bounding surface plasticity and critical state soil mechanics (Papadimitriou \& Bouckovalas, 2002). In fact, although the shear-volumetric coupling affects the residual changes of internal forces at the end of shaking in dry sand (Bilotta et al., 2014a, 2014b), this is less important for the prediction of the reversible changes of the internal forces generated in each cycle. Fig. 3 compares the maximum variation of bending moments provided by analyses in a cycle with both the above-mentioned constitutive models with centrifuge tests performed on dense and loose sand. Even though the experimental data are underestimated, the numerical results are in good agreement.

Soil models were calibrated according to the physical and mechanical properties reported in Table 1 . The unloadingreloading soil behaviour was defined by the hysteretic model provided by the Flac library and based on the well-known Masing rules (Masing, 1926). The decay of the normalised shear modulus with the increase of shear strain is introduced through the sigmoid function expressed in equation (1)

$$
G / G_{0}=\frac{m}{1+\exp \{-[\log (\gamma)-p] / n\}}
$$

Cyclic shear tests were numerically simulated on a cubic soil sample at different strain levels, from $\gamma=0.0001 \%$ to $\gamma=10 \%$, to calibrate the parameters $m, n$ and $p$ in equation (1) on the $G / G_{0}-\gamma$ and $D-\gamma$ curves obtained by Seed \& Idriss (1970). The parameters obtained for the two soil models are shown in Fig. 1(c), revealing that a good approximation of the mean target stiffness $(m=1, n=-0.5$ and $p=-1.4$ ) can lead to an excessive overestimation of the damping, due to the application of the Masing rules (Pelecanos et al., 2015). A more reasonable behaviour is predicted by the parameters $m=1, n=-0 \cdot 6$ and $p=-1 \cdot 2$, selected for the dynamic analyses.

The effect of the Mohr-Coulomb criterion on the hysteretic soil behaviour was evaluated by simulating cyclic shear tests under different values of confining stress $p^{\prime}=113 \mathrm{kPa}, p^{\prime}=320 \mathrm{kPa}, p^{\prime}=533 \mathrm{kPa}$ and $p^{\prime}=166 \mathrm{kPa}$,

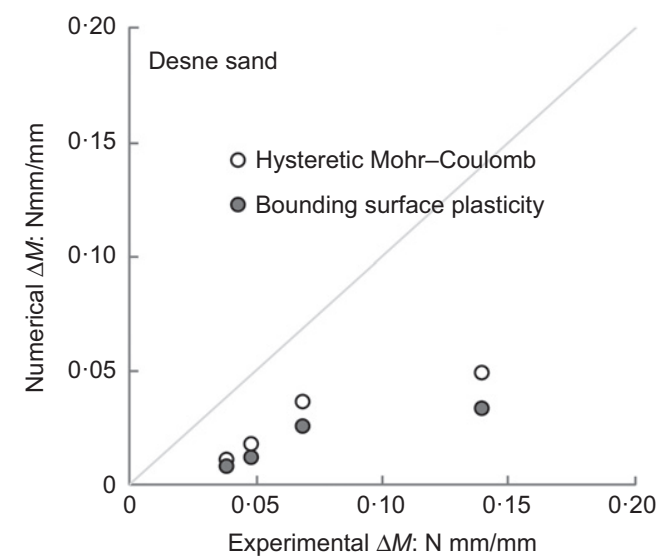

$p^{\prime}=333 \mathrm{kPa}, p^{\prime}=555 \mathrm{kPa}$, reproducing the lithostatic condition of loose and dense sand at a depth equal to $10 \mathrm{~m}, 30 \mathrm{~m}$ and $50 \mathrm{~m}$, respectively. The sensitivity analyses revealed that the introduction of the Mohr-Coulomb criterion implies the magnification of the shear stiffness degradation and the damping increase in correspondence of shear strain values higher than $0 \cdot 3 \%$ for the shallowest soil samples, while no differences are recognised at lower strain levels. Since shear strains higher than $0 \cdot 1 \%$ are expected to occur occasionally, for example under the peaks of input motions (see for instance Fig. 6, later), the cyclic behaviour is expected to be slightly influenced by the constitutive assumptions.

The low strain damping was introduced through the Rayleigh (1945) approach, assigning to the sand and bedrock layers the frequency-dependent damping ratio with the minimum $D_{0}=0.5 \%$ at the soil predominant frequency. The adopted $D_{0}$ value is consistent with the low strain damping provided by Seed \& Idriss (1970).

For the tunnel lining, typical values for a Class 25/30 concrete mix were adopted (BSI, 2004) and the damping $D_{0}$ was introduced through the Rayleigh (1945) approach, as had already been done for the soil low-strain damping.

It is worth noting how Kampas et al. (2019) previously reviewed a number of typical tunnel lining modelling approaches and the associated effect on axial forces and acceleration fields in the model of soil and lining pre- and post-cracking. A comparison between the results revealed that the lining axial forces and acceleration would not be significantly affected by the modelling approach. In this study, the structural material of the lining was assumed visco-elastic with the density, Young's modulus, Poisson's coefficient and damping ratio $D_{0}$ reported in Table 2 .

Accelerations and shear strain-time histories along the vertical sections V1, V2 and V3, and horizontal and vertical stresses in the mesh elements of the tunnel sections in Fig. 2 were recorded. The former allowed the dynamic behaviour of the soil-tunnel system to be identified and the latter were used to compute the variation of the internal forces induced by the seismic motion.

\section{FRAGILITY CURVES \\ Method}

The computation of fragility curves requires $(a)$ the definition of the engineering demand parameter, EDP, that measures the performance of the analysed system, and $(b)$ the choice of the seismic hazard IM. The EDP is defined by a threshold expressing the accepted damage level - that is

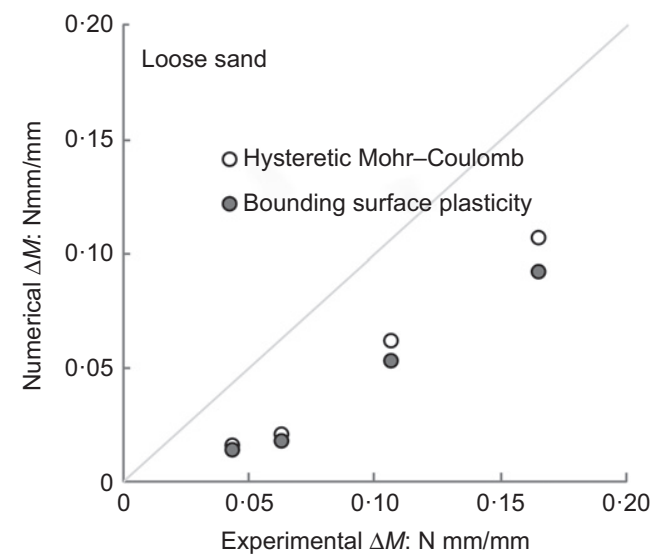

Fig. 3. Numerical plotted against experimental maximum cyclic change of bending moment obtained from centrifuge tests (modified after Conti et al. (2014)) 
the limit state - to compute the demand to capacity ratio, $\mathrm{DCR}_{\mathrm{LS}}$. The definition of such parameters and of their correlation depends on the failure mechanism expected to occur during the earthquake.

In this study, the cloud method was chosen to generate the fragility curves. Compared with the more commonly adopted stripe analyses, this method requires a lower number of seismic analyses to be carried out and it allows the adoption of unscaled records, thus avoiding the unrealistic and undesired modifications of scaled signals (Jalayer et al., 2015).

Moreover, in the studies of the tunnel seismic fragility available in the literature (e.g. Argyroudis \& Pitilakis, 2012; Argyroudis et al., 2017; Fabozzi et al., 2017), the probability of failure is computed as the probability of exceeding an IM value associated with the occurrence of the DS and a two-parameters fragility model is applied - that is the logarithm of the mean IM value and the standard deviation. The upgrade provided by the cloud method draws on the fact that the probability of failure is computed as the probability of exceeding the DCR $\mathrm{LS}_{\mathrm{L}}$ threshold on the basis of a lognormal distribution of $\mathrm{DCR}_{\mathrm{LS}}$, whose mean value is linearly dependent on IM in the logarithmic scale. Based on such hypotheses, the mean system performance at each IM and its standard deviation can be computed as follows in equations (2) and (3), respectively

$$
\begin{aligned}
\log \eta_{\mathrm{DCR}_{\mathrm{LS}} \mid \mathrm{IM}} & =\log (a)+b \log (\mathrm{IM}) \\
\sigma_{\log \mathrm{DCR}_{\mathrm{LS}} \mid \mathrm{IM}} & =\sqrt{\frac{\sum_{i=1}^{n}\left[\log (\mathrm{DCR} \mathrm{LSi})-\log \eta_{\left.\mathrm{DCR}_{\mathrm{LS}} \mid \mathrm{IM}_{\mathrm{i}}\right]^{2}}\right.}{N-2}}
\end{aligned}
$$

The vector of the parameters $\chi=\left[\log (a), b, \sigma_{\log \text { DCRLS|IM }}\right]$ is derived from the best fitting of the data $D=\left\{\left(\mathrm{IM}_{\mathrm{i}}, \mathrm{DCR}_{\mathrm{LSi}}\right)\right.$, $i=1: N\}$ resulting from the performed $N$ non-linear analyses. Equation (3) highlights that in the cloud method the uncertainties in the seismic performance are evaluated as the standard error in the estimation of the regression linking IM to DCR $_{\text {LS. }}$.

The probability of failure is then computed from the standard normal distribution shown in equation (4)

$$
P\left(\mathrm{DCR}_{\mathrm{LS}}>1 \mid \mathrm{IM}, \chi\right)=\left(\frac{\log \eta_{\mathrm{DCR}_{\mathrm{LS}} \mid \mathrm{IM}}}{\sigma_{\log \mathrm{DCR}_{\mathrm{LS}} \mid \mathrm{IM}}}\right)
$$

The regression predictions are reliable if the failure mechanism is mobilised in most of the dynamic analyses

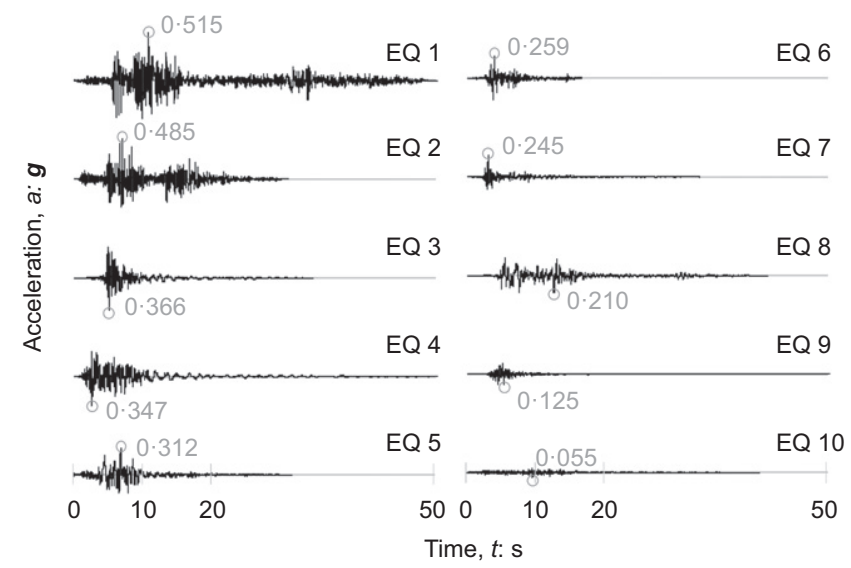

(a) performed. This condition is readily satisfied when the input motions are scaled to a different hazard level, as in the multiple stripe method. However, as already stated above, the use of unscaled natural records was preferred in this work, provided that most of the selected records were of sufficient magnitude to mobilise the investigated failure mechanism.

\section{Selected input motions}

Ten, moderate to severe, natural accelerograms were selected from the Pacific Earthquake Engineering Research Center (PEER) ground motion database to perform nonlinear dynamic analyses. Fig. 4 shows the ten natural accelerograms (Fig. 4(a)) and the associated acceleration response spectra (Fig. 4(b)).

The main characteristics of the selected signals are reported in Table 3: earthquake and station name, date, component, moment magnitude, $M_{\mathrm{W}}$, source-station epicentral distance, $R_{\text {epi }}$, equivalent shear wave velocity in the shallowest $30 \mathrm{~m}$ below the station, $V_{\mathrm{S} 30}$, PGA, peak ground velocity $(\mathrm{PGV})$ and predominant period, $T_{\mathrm{P}}$

Input motions should be selected to cover a large range of IM values, in order to reduce the standard error in the estimation of the regression between IM and $D_{C R}$. However, Jalayer et al. $(2015,2017)$ revealed that the improvement of the $\mathrm{DCR}_{\mathrm{LS}}-\mathrm{IM}$ regression prediction tends to reduce for more than ten simulations. Consequently, additional simulations were not performed, owing to the huge effort corresponding to the execution of the dynamic analyses. Consequently, the selection was based on the variability of PGA, PGV and spectral accelerations associated with the first resonance period of the soil ( $T_{1}$ in Fig. 4(b)), as well as of the frequency content, as proven by the variable predominant period, $T_{\mathrm{B}}$ in Table 3 . Furthermore, the $V_{\mathrm{S} 30}$ in Table 3 proves that the selected signals were recorded on sufficiently stiff soil, identified as A or B type in the Eurocode, thus avoiding the possible signal modifications induced by local site effects. A Butterworth fourth-order filter was applied to all signals to limit their frequency content to the range $0 \cdot 1-25 \mathrm{~Hz}$.

\section{Soil response and site effects under non-linear dynamic analyses}

The initial stress conditions were reproduced into two steps.

(a) Computation of the lithostatic stress acting in free-field conditions.

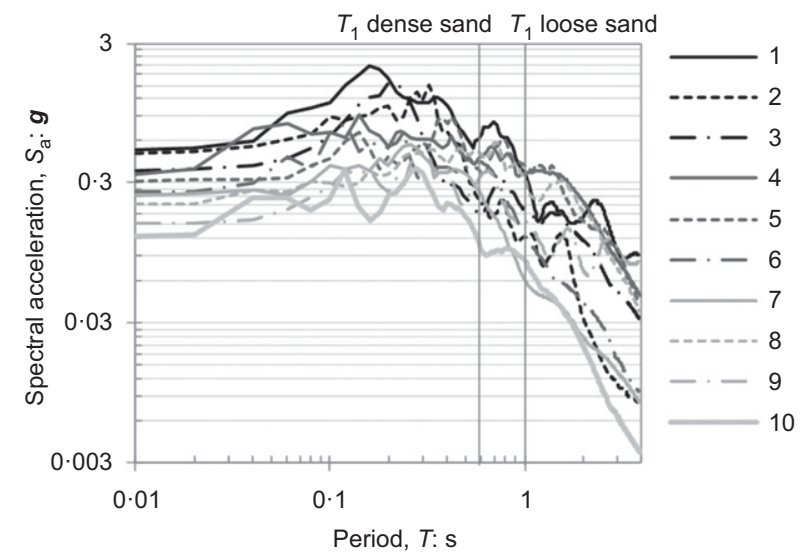

(b)

Fig. 4. Acceleration (a) time histories and (b) response spectra of the selected input motions. Labels superimposed on (b) refer to the predominant period $T_{1}$ of dense sand and loose sand 
Table 3. Main characteristics of the selected signals

\begin{tabular}{|c|c|c|c|c|c|c|c|c|c|c|}
\hline & Earthquake name & Station name & Date & Component & $M_{\mathrm{W}}$ & $\begin{array}{c}R_{\text {epi }}: \\
\mathrm{km}\end{array}$ & $\begin{array}{c}V_{\mathrm{S} 30}: \\
\mathrm{m} / \mathrm{s}\end{array}$ & PGA: $g$ & $\begin{array}{l}\mathrm{PGV} \text { : } \\
\mathrm{cm} / \mathrm{s}\end{array}$ & $T_{\mathrm{P}}: \mathrm{s}$ \\
\hline 1 & Manjil, Iran & Abbar & $21 / 06 / 1990$ & NS & $7 \cdot 4$ & $21 \cdot 75$ & 723 & $0 \cdot 515$ & $39 \cdot 00$ & $0 \cdot 16$ \\
\hline 2 & Duzce & AI_156_VO & 19/11/1999 & EW & $7 \cdot 1$ & $27 \cdot 17$ & 482 & $0 \cdot 485$ & $16 \cdot 00$ & $0 \cdot 32$ \\
\hline 3 & Centro Italia & Norcia NRC & $24 / 08 / 2016$ & NS & $6 \cdot 0$ & $15 \cdot 30$ & 687 & $0 \cdot 366$ & 18.69 & $0 \cdot 22$ \\
\hline 4 & L'Aquila & V. Aterno & $06 / 04 / 2009$ & NS & $6 \cdot 3$ & 1.80 & 705 & $0 \cdot 347$ & $38 \cdot 43$ & $0 \cdot 14$ \\
\hline 5 & Kobe, Japan & Kobe University & $17 / 01 / 1995$ & EW & $6 \cdot 9$ & $25 \cdot 40$ & 1043 & $0 \cdot 312$ & $30 \cdot 88$ & $0 \cdot 42$ \\
\hline 6 & Greece & ITSAK & 07/09/1999 & EW & $5 \cdot 9$ & $19 \cdot 83$ & 547 & $0 \cdot 259$ & $17 \cdot 18$ & $0 \cdot 12$ \\
\hline 7 & Parkfield-02 CA & Parkfield & $9 / 28 / 2004$ & EW & $6 \cdot 0$ & $9 \cdot 27$ & 906 & $0 \cdot 245$ & $13 \cdot 19$ & $0 \cdot 26$ \\
\hline 8 & Balkan Peninsula & Ulcinj-Albatros & $15 / 04 / 1979$ & EW & $6 \cdot 9$ & $19 \cdot 72$ & $A^{*}$ & $0 \cdot 210$ & $26 \cdot 78$ & $0 \cdot 72$ \\
\hline 9 & Cosenza & Mormanno MRM & $25 / 10 / 2012$ & NS & $5 \cdot 2$ & $2 \cdot 40$ & 1906 & $0 \cdot 125$ & $8 \cdot 10$ & 0.28 \\
\hline 10 & Chi-Chi, Taiwan & CHY101 & 21/09/1999 & NS & $6 \cdot 2$ & $44 \cdot 71$ & 804 & $0 \cdot 050$ & $2 \cdot 66$ & $0 \cdot 08$ \\
\hline
\end{tabular}

A* indicates that soil station is identified as rigid, but no field investigations were performed to verify its stiffness.

(b) Solution of the equilibrium of the wished-in-place tunnel, neglecting the effects of the excavation process on its mechanical behaviour. In detail, soil elements were switched into concrete elements where the lining was located, and simultaneously null mechanical properties were assigned to soil elements inside the lining to reproduce the void.

The identification of the soil dynamic behaviour and of the modifications induced by the tunnel excavation was evaluated first. Thus, a white noise signal lasting $30 \mathrm{~s}$, having a flat spectrum in the range of frequencies $0 \cdot 1-25 \mathrm{~Hz}$, was applied in the form of a shear-stress time history at the bottom of the analysis domain. Compared with the more common application of a wavelength, the use of white noise is more suitable to identify the dynamic behaviour in complex conditions such as a system of cavities (de Silva \& Scotto di Santolo,
2018) and soil-structure coupled systems (de Silva et al., 2018).

The soil amplification functions were computed along the three vertical sections $\mathrm{V} 1, \mathrm{~V} 2$ and $\mathrm{V} 3$ as the ratio between the amplitude of the fast Fourier transform of the accelerationtime histories recorded at the ground level and on the top of the bedrock layer. Fig. 5 shows the results before ('Full' in the key of Fig. 5) and after ('Void' in the key of Fig. 5) the excavation of the shallow (Fig. 5(a)) and deep (Fig. 5(b)) tunnels in loose and dense sand. Independently of the reference scheme, the amplification functions of the full models are close together along the three vertical sections, with the first resonant frequency equal to $1 \mathrm{~Hz}$ and $1.7 \mathrm{~Hz}$ for loose and dense sand, respectively. The numerical models are validated by the computed frequency values, which are very close to the theoretic predominant frequencies of a layer in which the stiffness increases with depth (Dobry et al.,
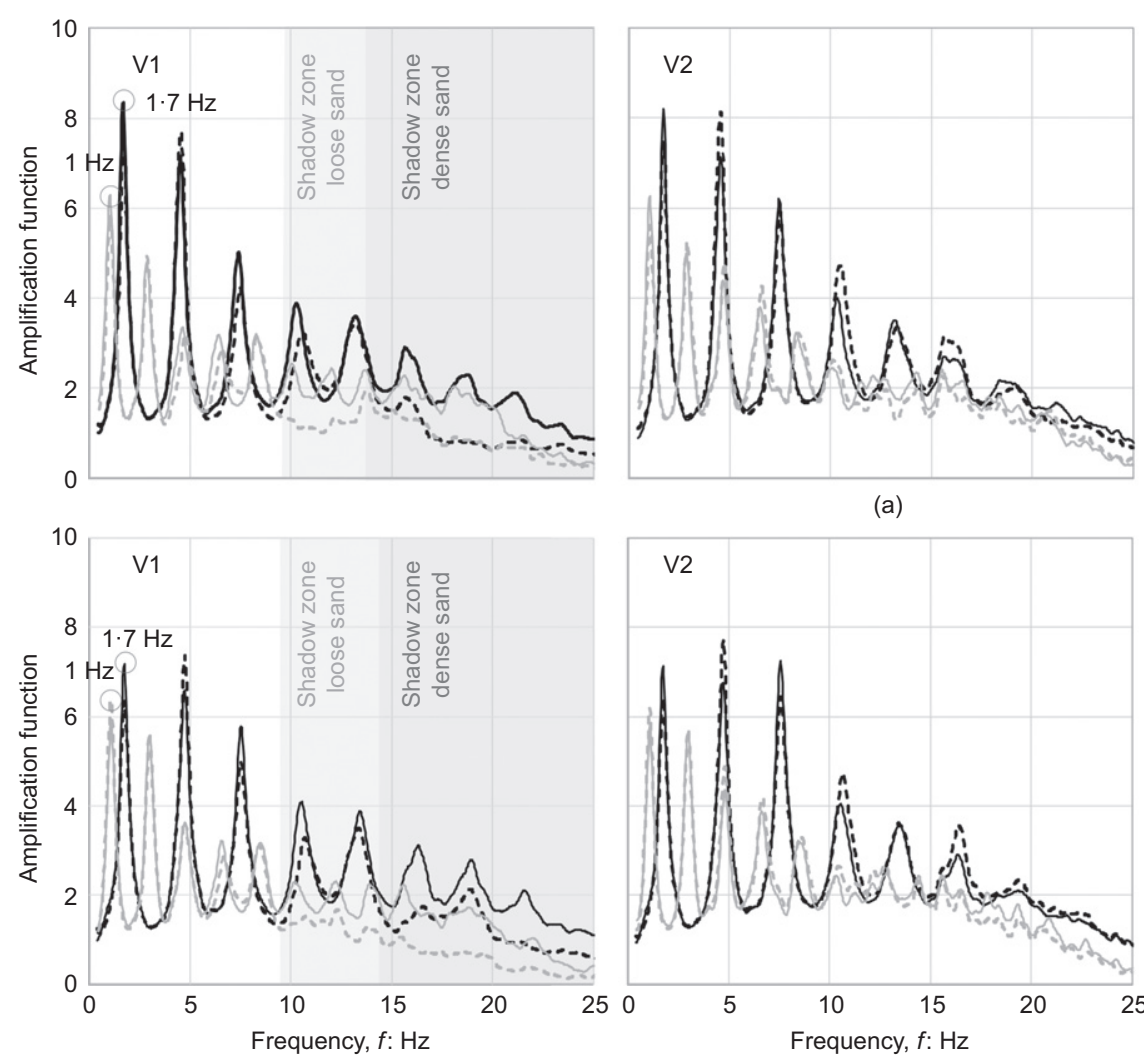

(a)

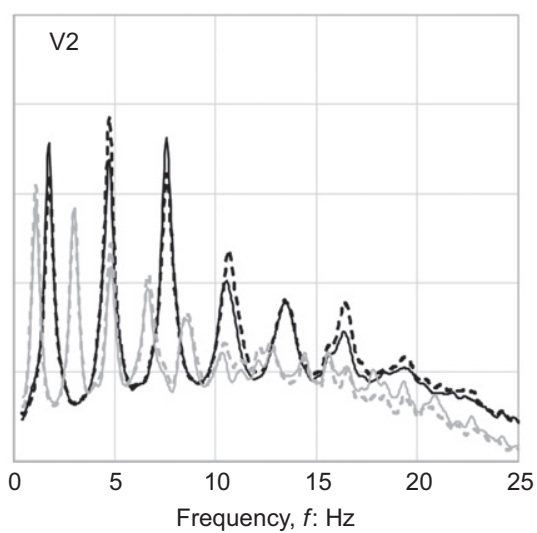

(b)
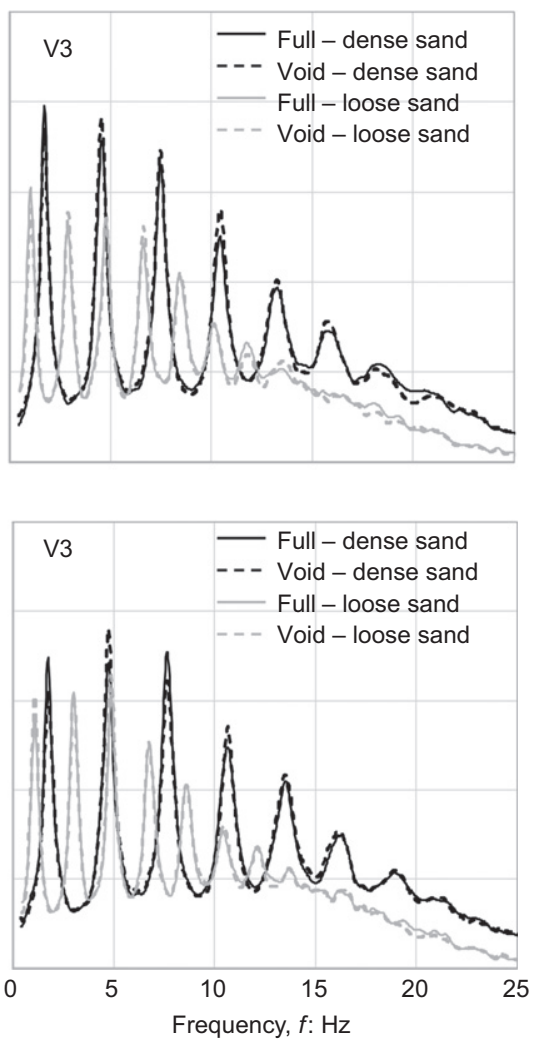

Fig. 5. Amplification functions computed along the vertical sections V1, V2 and V3, before (full) and after (void) the excavation of the (a) shallow and (b) deep tunnel 
1976), resulting in $f=1.08 \mathrm{~Hz}$ and $f=1.68 \mathrm{~Hz}$ for loose and dense soil, respectively.

The peaks associated with the predominant frequencies are not affected by the presence of the tunnel, nor is the whole dynamic response along the vertical sections V2 and V3. On the other hand, a drop at high frequencies is recognised along the vertical V1 with respect to the amplification functions computed before the excavation (dashed lines in Fig. 5). The shadow zone of loose and dense sand is, respectively, limited by a frequency $f_{\min } \approx 9 \mathrm{~Hz}$ and $f_{\min } \approx 14 \mathrm{~Hz}$, independently of the tunnel depth. Since the attenuation of the motion occurs at moderate to high frequency, near-fault ground motions are expected to be reduced by the presence of the tunnel. On the contrary, smaller effects are expected at the far field due to the loss of the high-frequency content of the propagated signal.

Figure 6(a) summarises the results of the non-linear dynamic analyses in terms of amplification factors - that is the ratio between the PGA computed at the ground level $\left(\mathrm{PGA}_{\mathrm{gl}}\right)$ and on the top of the bedrock layer $\left(\mathrm{PGA}_{\mathrm{im}}\right)$. Independently of the reference scheme, the amplification factor reduces when increasing the amplitude of the input motion, due to the soil hysteretic behaviour. As expected, the effect of non-linearity is so pronounced that an attenuation of the seismic motion is observed in the loose sand under the strongest five earthquakes. Even though the same decay of shear stiffness (see Fig. 1(c)) was applied to the soil profiles, no attenuation of the seismic motion is observed in the dense sand, due to its higher initial stiffness. As an example, the contours of the maximum shear strain, $\gamma_{\max }$, developed around $\mathrm{V} 1$ under the earthquake number 1 are shown in
Figs 6(b)-6(e), demonstrating that the shear strains are much larger and more diffused in the loose sand models.

Linear regressions were fitted through the computed amplification factors along the three control vertical sections as shown in Fig. 6, for an easy comparison of the different behaviours. In most of the cases for a deep tunnel, the highest amplifications occur along the vertical V2 (light grey continuous line). When the tunnel is deep and the sand is dense, the presence of a void zone (i.e. V1 - dashed line) reduces the ground motion with respect to the free-field results (V3 - continuous black line). This trend most probably confirms the 'shadowing effect' observed under the low-amplitude excitation (see Fig. 5 in the earlier section entitled 'Numerical models'), due to the loss of highfrequency content when the seismic wave crosses the tunnel.

A different trend is recognised for the shallow tunnel in dense sand for all PGAs and in loose sands for PGA values greater than $0 \cdot 3 \boldsymbol{g}$ approximately. In these cases, the maximum amplifications correspond to V1 due to soil non-linear behaviour. As evidenced by the contours shown in Figs 6(b) and 6(c), the higher strain level mobilised around the lining reaches the ground level only when the tunnel is shallow. As a consequence, the ground motion is differently modified depending on the co-existence between the 'shadowing effect' of void and the soil non-linearity.

\section{Earthquake-induced forces in the lining}

During the seismic analyses, the stresses acting in correspondence to the tunnel lining control sections

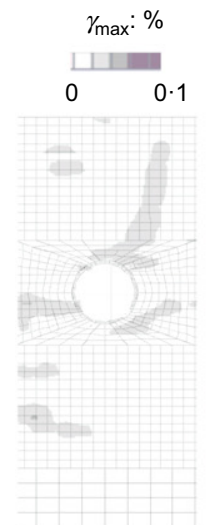

(b) $\gamma_{\max }: \%$

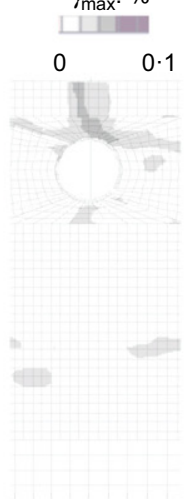

(c)
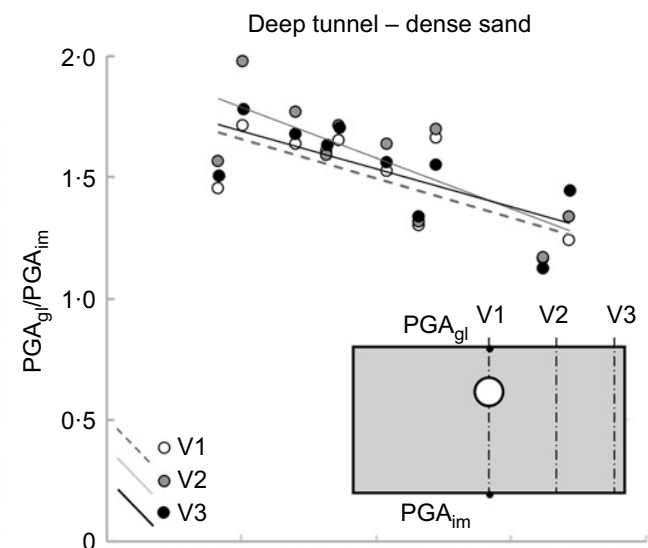

Shallow tunnel - dense sand
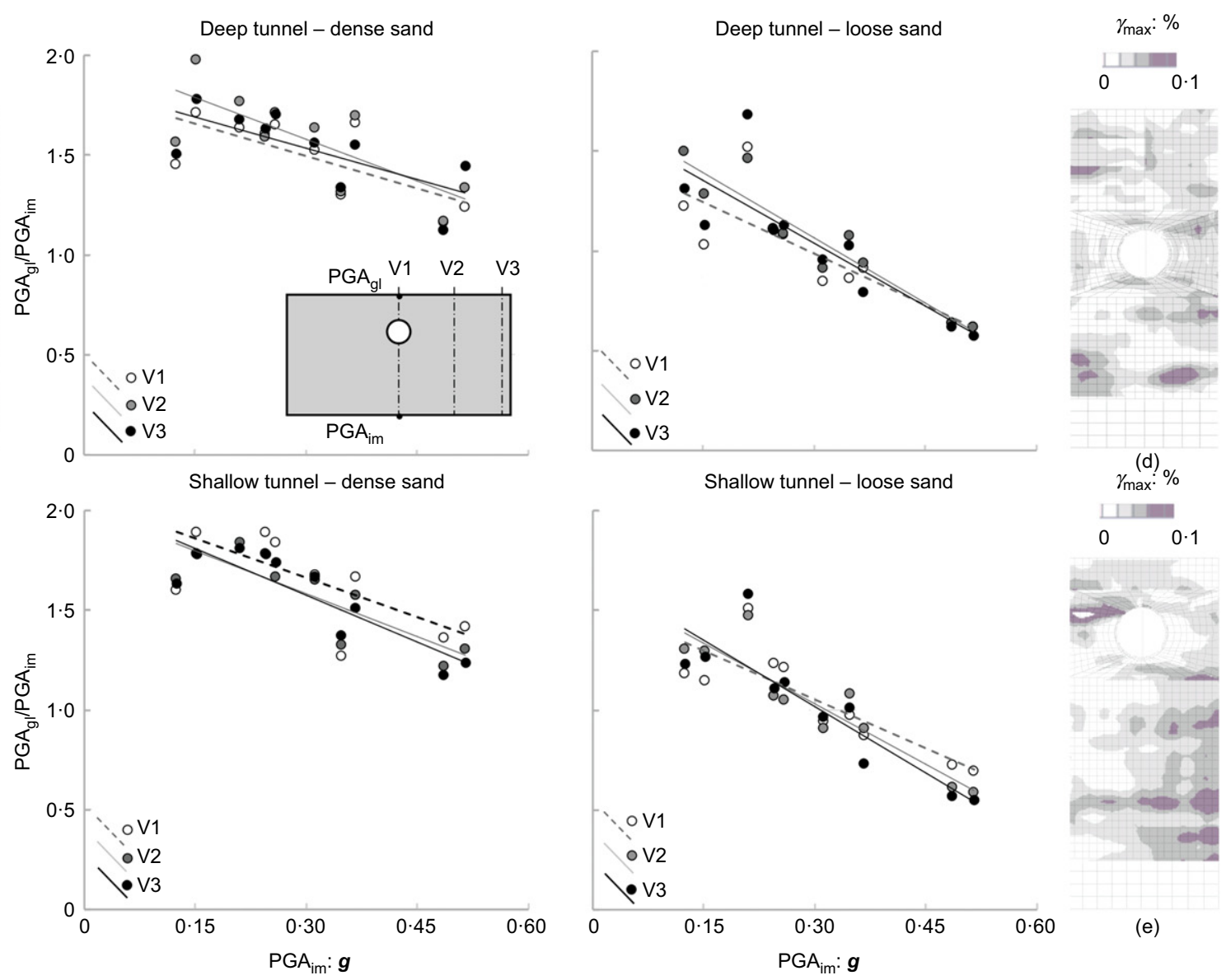

0 $0 \cdot 1$

(a)

Fig. 6. (a) Comparison among the PGA amplification factors computed along the vertical sections V1, V2 and V3; (b)-(e) contours of maximum shear strain under earthquake no. 1 
identified in Fig. 2 were recorded to compute the evolution of axial force and the bending moment. Fig. 7 compares the static load (black lines) with the maximum and minimum profiles (grey lines) induced by each earthquake in the lining of the four reference schemes. In all cases, the maximum variation of the internal forces is recognised in sections $45^{\circ}$, $135^{\circ}, 225^{\circ}$ and $315^{\circ}$, with significant positive and negative peaks in the bending moment. This trend is indicative of the expected ovalisation of the cross-section under the exciting cyclic shear strain.

A larger increase of internal forces affects deep tunnels, in turn affecting the safety. Significant reductions of the axial forces are shown in shallow tunnels, critically approaching zero. Since the bending resistance typically increases with axial force, its reduction influences the tunnel safety.

These numerical results have been compared with the experimental results of the centrifuge tests carried out by Lanzano et al. (2012) and, when possible, with their numerical interpretations available in the literature. Fig. 8 for instance, shows the comparison in terms of maximum cyclic change of bending moment, $\Delta M_{\max }$, per unit length of tunnel lining, reached during the seismic excitation at the most loaded tunnel sections, as a function of the peak value of the input time history of acceleration applied at the base of the model, PGA $\mathrm{im}_{\text {. }}$. Fig. 8(a) refers to the case of shallow tunnel $(C / D=1)$, for dense and loose sand, corresponding to T1 and T2 models Lanzano et al. (2012), while Fig. 8(b) concerns the case of deep tunnel $(C / D=2)$ for both dense and loose sand cases, corresponding to $\mathrm{T} 3$ and $\mathrm{T} 4$ centrifuge models in Lanzano et al. (2012), respectively. In the figure,
$\Delta M_{\max }$ has been made non-dimensional by dividing it by $\left(\tau_{\mathrm{eq}} r^{2}\right)$, where $r$ is the radius of the tunnel and $\tau_{\mathrm{eq}}$ is the equivalent shear stress. The latter is representative of the shear stress transmitted to the tunnel lining during shaking and it was calculated from the horizontal equilibrium of a soil column undergoing inertial forces through equation (5): $a_{\mathrm{cc}, \max }$ is the maximum acceleration at the mid-depth of the tunnel axis; $\boldsymbol{g}$ is acceleration due to gravity; $\gamma_{\text {soil }}$ is the unit weight of soil; and $H_{\text {axis }}$ is the depth of the tunnel axis.

$$
\tau_{\mathrm{eq}}=\frac{a_{\mathrm{cc}, \max }}{\boldsymbol{g}} \gamma_{\mathrm{soil}} H_{\mathrm{axis}}
$$

The experimental data of the deepest tunnel models (T3 and T4 tests) were made available to the scientific community through the round robin tunnel test (RRTT) organisation (Bilotta et al., 2014a, 2014b). Consequently, multiple authors predicted the dynamic behaviour of the model tunnels (i.e. Amorosi et al., 2014; Conti et al., 2014; Gomes, 2014; Hleibieh et al., 2014; Tsinidis et al., 2014; Abate et al., 2015; Fabozzi \& Bilotta, 2016; Tsinidis et al., 2016; Ochmański et al., 2019). Different constitutive soil models and numerical codes were adopted.

The numerical results shown in Fig. 8(b) represent, as a function of the corresponding $\mathrm{PGA}_{\mathrm{im}}$, the mean values of the $\Delta M_{\max }$ calculated by Amorosi et al. (2014), Conti et al. (2014), Gomes (2014), Hleibieh et al. (2014) and Tsinidis et al. (2014), in both blind predictions and back-analyses. The range of variability of the numerical results is also illustrated in Fig. 8.
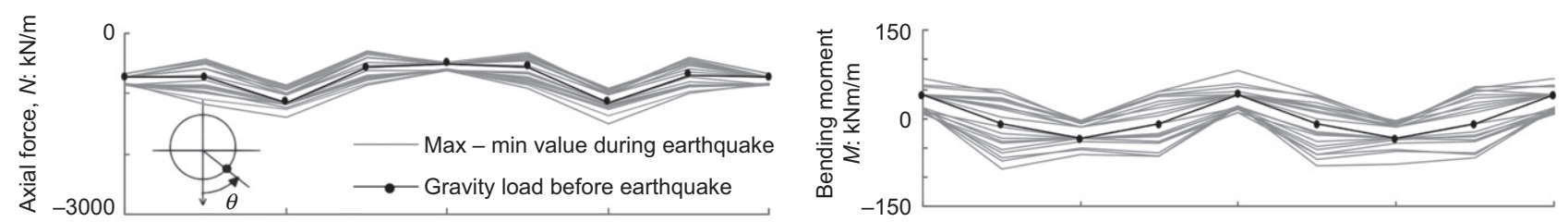

(a)
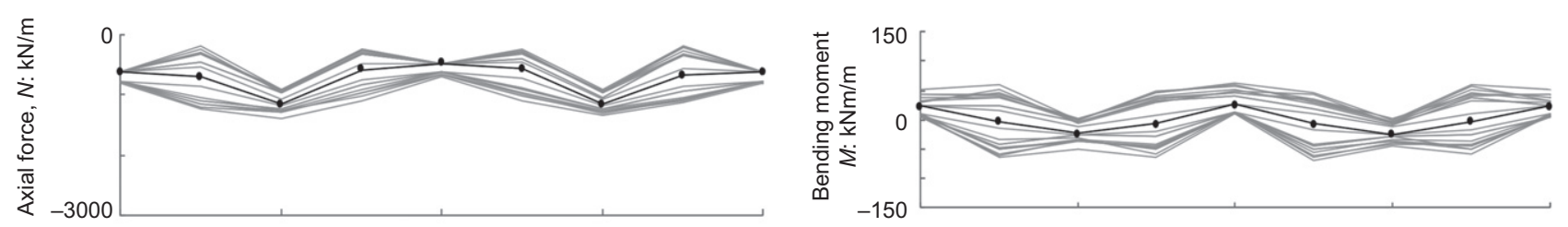

(b)
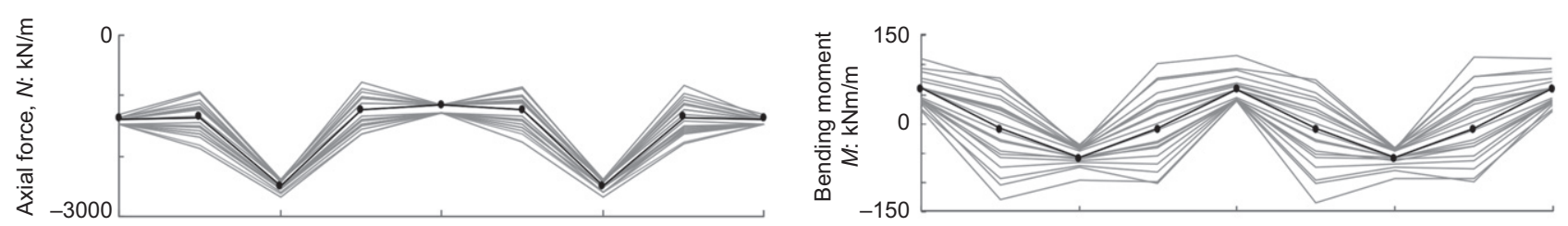

(c)
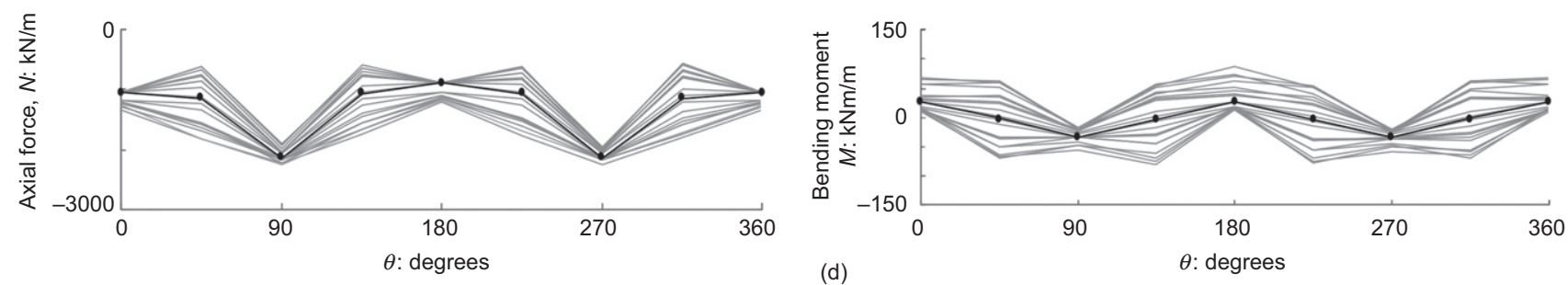

Fig. 7. Envelope of axial force and bending moment induced in the lining by each earthquake: (a) shallow tunnel - loose sand; (b) shallow tunnel dense sand; (c) deep tunnel - loose sand; (d) deep tunnel - dense sand 

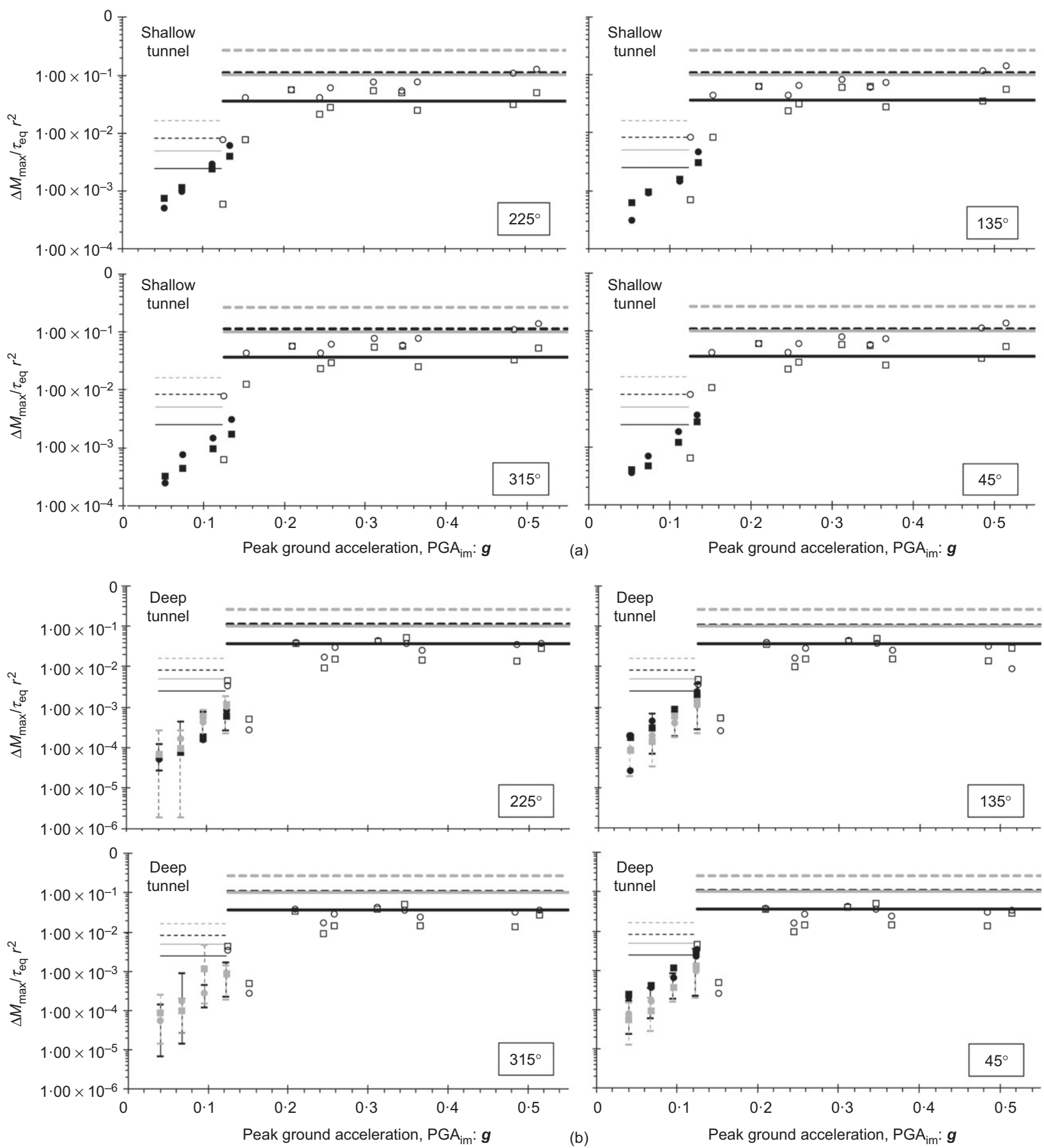

\begin{tabular}{|c|c|c|c|c|c|}
\hline & \multicolumn{3}{|c|}{ Centrifuge tests } & \multicolumn{2}{|r|}{ Present study } \\
\hline & Experimental $\left(^{*}\right)$ & Numerical $\left({ }^{* *}\right)$ & Wang solution (1993) & Numerical & Wang solution (1993) \\
\hline Dense sand & ○ & $\vdash-1$ & $\begin{aligned} F & =f\left(G_{0}\right) \\
F & =f\left(G_{\mathrm{min}}^{\mathrm{mob}}=0 \cdot 3 G_{0}\right)\end{aligned}$ & $\mathrm{O}$ & $\begin{aligned} F & =f\left(G_{0}\right) \\
\text { D-n-- } F & =f\left(G_{\min }^{\mathrm{mob}}=0 \cdot 3 G_{0}\right)\end{aligned}$ \\
\hline Loose sand & $\mathbf{\square}$ & $1-\square--1$ & $\begin{aligned} F & =f\left(G_{0}\right) \\
F & =f\left(G_{\mathrm{min}}^{\mathrm{mob}}=0.3 G_{0}\right)\end{aligned}$ & $\square$ & $\begin{aligned} F & =f\left(G_{0}\right) \\
-----F & =f\left(G_{\mathrm{min}}^{\mathrm{mob}}=0 \cdot 3 G_{0}\right)\end{aligned}$ \\
\hline
\end{tabular}

Fig. 8. Comparison of the maximum cyclic change of bending moment resulting from this study for the (a) shallow and (b) deep tunnel with the experimental ones from centrifuge tunnel tests performed by Lanzano et al. (2012) and corresponding numerical predictions $(*$ signifies experimental results found in the paper by Lanzano et al. (2012); ** signifies the mean value of numerical interpretations of centrifuge tests found in the papers by Amorosi et al. (2014), Gomes (2014), Hleibieh et al. (2014) and Tsinidis et al. (2014))

In Figs 8(a) and 8(b), the analytical solutions of Wang (1993) (which extend Hoeg (1968)) are also shown, assuming equation (6)

$$
\frac{\Delta M_{\max }}{\tau_{\text {eq }} r^{2}}=\frac{12\left(1-v_{\text {soil }}\right)}{6 F+15-18 v_{\text {soil }}}
$$

where to consider the non-linear soil stiffness decay during shaking, the flexibility ratio, $F$, is calculated as in equation (7)

$$
F=\frac{G_{\text {soil,mob }}\left(1-v_{\text {lining }}^{2}\right) D_{\text {tunnel }}^{3}}{24(E I)_{\text {lining }}}
$$


where $G_{\text {soilmob }}$ is the mobilised shear modulus that changes case by case and, for the sake of simplicity, is assumed either equal to its maximum value, $G_{0}$ (continuous line), which provides a low estimate, or equal to a conventional value of $0 \cdot 3 G_{0}$, which provides a realistic estimation of the highest possible value in all the cases (dashed line).

Despite the influence of soil plasticity on the accumulation of residual bending moments at the end of shaking (experimentally confirmed by Cilingir \& Madabhushi (2011a) and Lanzano et al. (2012)), the comparison shown in Fig. 8 of numerical predictions (that include different plasticity models) with the classical Wang's solutions (that assume an equivalent linear behaviour) is legitimate since the calculated steady-state cyclic changes of bending moments occurring during shaking are considered.

The analytical solution largely overestimates the centrifuge tests results (black markers) and the associated numerical predictions (grey markers). This is expected, according to Kontoe et al. (2014), considering that the tunnel lining tested in the centrifuge was extremely flexible ( $F$ ranges between 644 (dense sand) and 322 (loose sand) for the initial shear modulus $G_{0}$, and between 193 (dense sand) and 96 (loose sand) for the mobilised shear modulus $0 \cdot 3 G_{0}$ ).

The numerical results of this study (empty markers), that take into account stiffer linings ( $F$ ranges between 37 (dense sand) and 11 (loose sand) for $G_{0}$, and between 11 (dense) and 3 (loose sand) for $0 \cdot 3 G_{0}$ ), are closer to the analytical solutions, and in many cases fall within their observed range.

\section{Discussion of results}

For strategic linear infrastructures, minor damage should be prevented and, hence, the fragility curves of tunnels were computed with reference to the first onset of cracks in the lining. Such a limit state is associated with reaching the concrete tensile strength, $\sigma_{\mathrm{t}}$ (see Table 2). The resistance was consequently calculated as the moment $\left(M_{\text {res }}(t)\right.$ in Fig. 9(a)) induced in the resistant section by the stress diagram plotted in Fig. 9(a), in which $\sigma_{\mathrm{c}}$ is the maximum compression stress acting in the section, while $\sigma_{\text {ru }}$ and $\sigma_{\mathrm{rl}}$ is the stress acting in the upper and lower steel reinforcements, respectively. Since the response of the structural materials is still elastic at the onset of the assumed limit state, $\sigma_{\mathrm{ru}}$ and $\sigma_{\mathrm{rl}}$ are typically normalised through the homogenisation coefficient $n_{\mathrm{c}}=6 \cdot 7$, calculated from the ratio between the steel and concrete Young's moduli. All the stress values can be easily calculated from geometric considerations, once the depth of the neutral axis $(x(t)$ in Fig. 9(a)) is known from assuming equilibrium with the axial force $N(t)$. As observed in the section 'Soil response and site effects under non-linear dynamic analyses', the axial force changes during the seismic excitation, hence the resistant moment $M_{\text {res }}(t)$ is also time dependent. This is a critical aspect of the seismic tunnel performance considered in this study, where the static equilibrium was solved at each time step using a routine implemented in Matlab. As an example, the evolution of $M_{\text {res }}(t)$ under earthquake number 1 for the shallow tunnel in loose soil is compared with the loading moment $M_{\text {load }}(t)$ in Fig. 9(b); for the sake of clarity, the negative resistant moment is omitted, as it is equal and

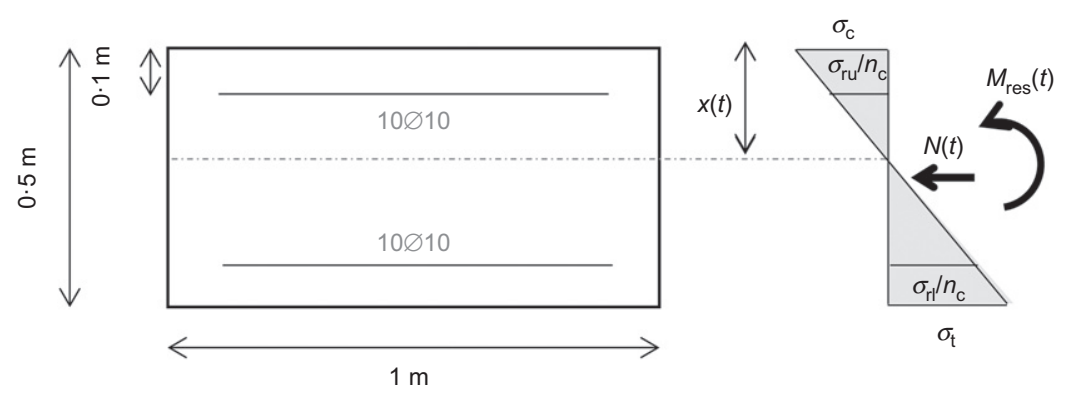

(a)
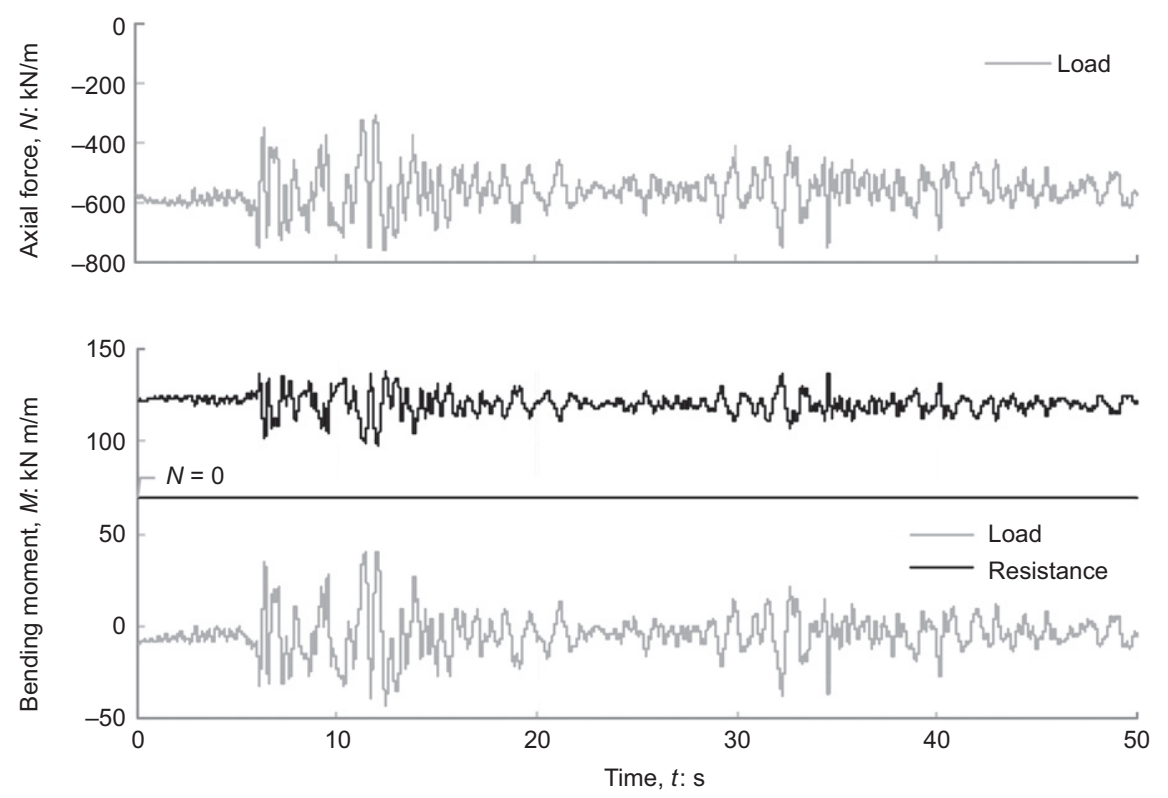

(b)

Fig. 9. Scheme of the tunnel section for (a) the calculation of the resistance moment and (b) the evolution with time of the axial force and load and resistance moments in section $135^{\circ}$ of shallow tunnel in loose sand under earthquake number 1 
opposite to the positive one due to the symmetry of the resistant section. As expected, the numerous and instantaneous decreases of $N(t)$ reduce the resistant moments and, in most cases, the lowest values occur when the loading moment shows the most significant peaks.

The seismic performance of the tunnel was 'measured' by the maximum ratio between the loading and the resistance moment - that is the maximum mobilised strength during earthquake

$$
\mathrm{DCR}_{\mathrm{LS}}=\max \left[\frac{M_{\text {load }}(t)}{M_{\mathrm{res}}(t)}\right]
$$

Since the bending loads are responsible for the tunnel damage, demand and capacity are generally identified with the loading and resisting bending moment. In the existing studies on the fragility of tunnels (e.g. Argyroudis \& Pitilakis, 2012; Argyroudis et al., 2017), only the peak loading values are assumed in the calculation of the DCR $\mathrm{LS}$ and the evolution of the capacity associated with the variation of the axial load during the whole excitation is neglected.

Table 4 reports the mean values and the standard deviations, $\sigma$, computed in the four tunnel sections $\left(45^{\circ}\right.$, $135^{\circ}, 225^{\circ}$ and $315^{\circ}$ ), in which the highest increment of internal forces was recognised during the dynamic analyses (see Fig. 7). In all cases, $30 \%$ of strength is mobilised on average, with values exceeding $40 \%$ in the case of a shallow tunnel in dense sand.

Interpreting the bending moment as an EDP, the limit state was assumed to be reached when $\mathrm{DCR}_{\mathrm{LS}}=1$. Thus, the probability of failure was calculated through the cloud method (see the section entitled 'Method'), assuming the PGA and $P G V$ of the input motion $\left(\mathrm{PGA}_{\mathrm{im}}\right.$ and $\left.\mathrm{PGV}_{\mathrm{im}}\right)$ and recorded at the surface in free-field conditions $\left(\mathrm{PGA}_{\mathrm{gl}}\right.$ and $\mathrm{PGV}_{\mathrm{gl}}$ ), as seismic IMs.

Even though the numerical fragility curves available in literature assume the PGA of the ground surface as IM, the

Table 4. Mean value and standard deviation of mobilised resistance $D_{C R}$

\begin{tabular}{|c|c|c|c|c|c|c|c|c|}
\hline & \multicolumn{2}{|c|}{$45^{\circ}$} & \multicolumn{2}{|c|}{$135^{\circ}$} & \multicolumn{2}{|c|}{$225^{\circ}$} & \multicolumn{2}{|c|}{$315^{\circ}$} \\
\hline & Mean: $\%$ & $\sigma: \%$ & Mean: $\%$ & $\sigma: \%$ & Mean: $\%$ & $\sigma: \%$ & Mean: $\%$ & $\sigma: \%$ \\
\hline Deep tunnel_dense sand & 28 & 16 & 27 & 13 & 26 & 13 & 30 & 16 \\
\hline Deep tunnel_loose sand & 29 & 15 & 32 & 19 & 31 & 16 & 32 & 20 \\
\hline Shallow tunnel_dense sand & 39 & 15 & 38 & 10 & 35 & 10 & 43 & 16 \\
\hline Shallow tunnel_loose sand & 32 & 12 & 32 & 12 & 34 & 11 & 32 & 14 \\
\hline
\end{tabular}

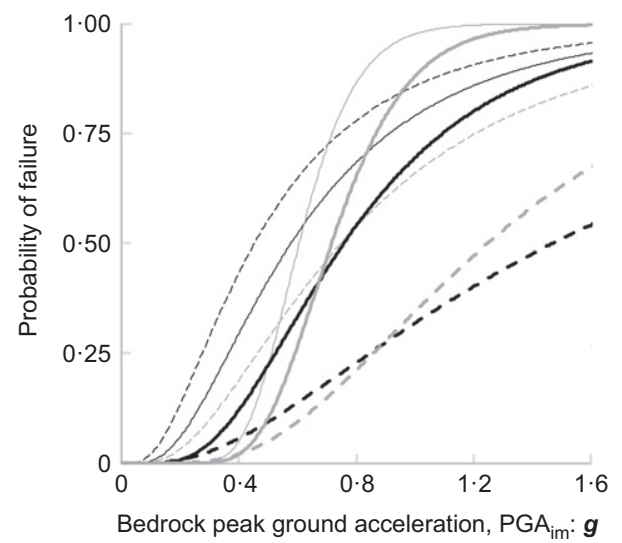

(a)

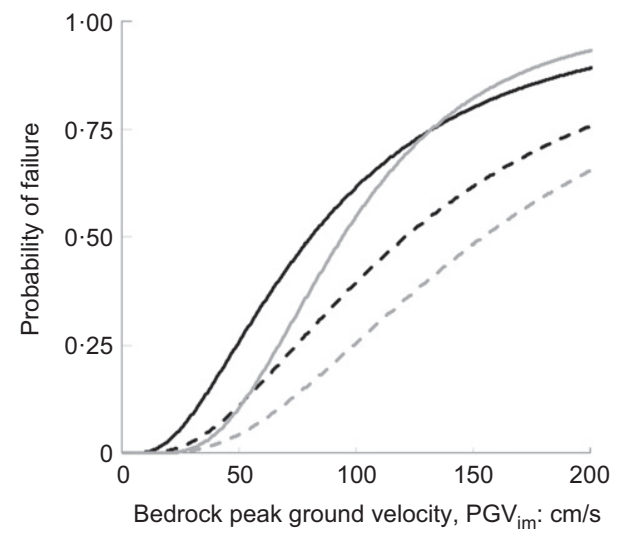

(c)

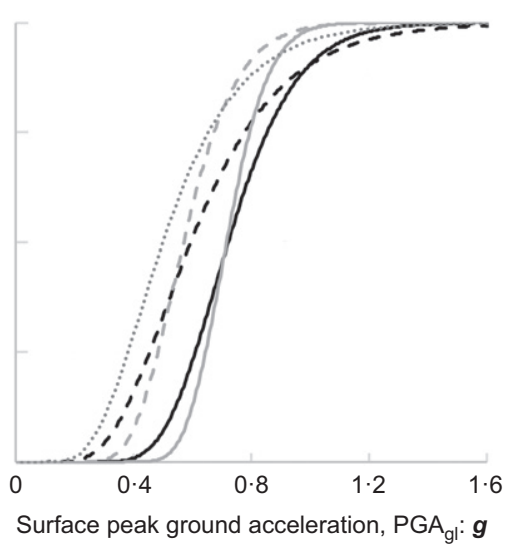

(b)

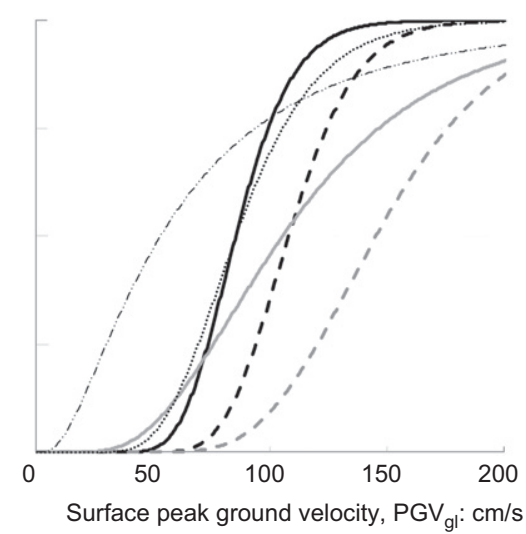

(d)

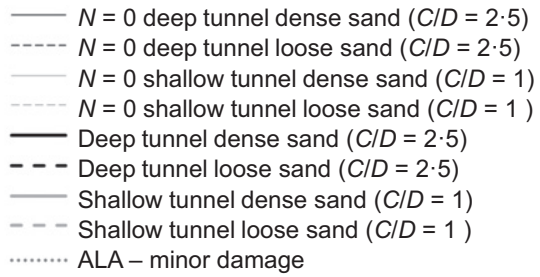

Fig. 10. Numerical fragility curves for shallow and deep tunnel in loose and dense sand proposed in this study and comparison with the empirical one expressed as a function of: (a) the PGA of the input motion (i.e. of a stiff and flat rock outcrop); (b) the PGA at the ground level (i.e. accounting for the site effects); (c) the PGV of the input motion; (d) the PGV at the ground level 


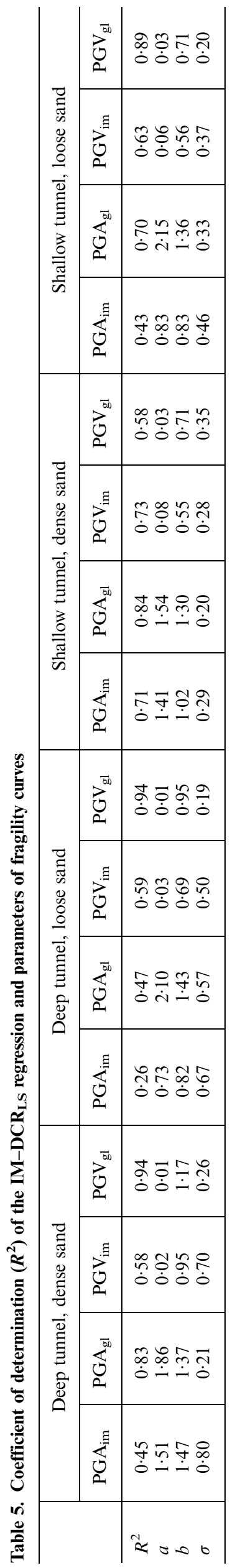

correlation of the demand-to-capacity ratio was checked, in addition

(a) against the PGV at the surface, because the ovalisation of the transverse section of the tunnel lining is governed by the shear strains close to the tunnel, which is correlated to the soil ground velocity

(b) against the PGA and PGV of the input motion, because fragility curves expressed as a function of the input motion parameter are more useful for real-time analyses, since signals provided by the seismic stations are recorded on stiff rock outcrop and consequently do not include site effects.

The envelopes of the fragility curves computed for the control sections of each reference scheme are shown in Fig. 10 as a function of the PGA (Fig. 10(a)) and PGV (Fig. 10(c)) of the input motion (i.e. of a stiff and flat rock outcrop) and of the PGA (Fig. 10(b)) and PGV (Fig. 10(d)) at the ground level (i.e. accounting for the site effects). Table 5 reports the determination coefficient of the $\mathrm{DCR}_{\mathrm{LS}}-\mathrm{IM}$ regression in the logarithmic scale together with the three parameters of the fragility model.

As testified by its highest $R^{2}$ values, $\mathrm{PGV}_{\mathrm{gl}}$ captures the relevant features affecting the seismic performance of soiltunnel systems.

When the parameters of the input motion PGA $_{\mathrm{im}}$ and $\mathrm{PGV}_{\text {im }}$ are assumed as IMs, there is a scatter between the probability of failure of tunnels in dense and loose sand associated with the different site amplifications. In fact, high amplitudes of input motion are amplified by dense sand, but they are dampened by loose soil (see Fig. 6), thus the seismic loads acting in the lining are reduced and, consequently, result in a lower fragility of the tunnel embedded in loose sand. As a matter of fact, the scatter is evidently reduced, when the curves are expressed in terms of ground motion parameters $\mathrm{PGA}_{\mathrm{gl}}$ and $\mathrm{PGV}_{\mathrm{gl}}$. If the occurring earthquake is strong enough to produce the same high amplitudes at the ground level of the different soil profiles, damage is expected to tunnels in dense as well as in loose soil.

The computation of fragility curves in terms of $\mathrm{PGA}_{\mathrm{im}}$ was repeated assuming zero axial force acting in the lining and consequently the lowest bending resistance of the section, which is superimposed on Fig. 8 through the horizontal line. The resulting curves are plotted using thinner lines in Fig. 10, confirming the beneficial effect of the axial forces in the reduction of the fragility.

The reliability of the numerical curves was checked through comparison with empirical fragility curves generated from the damage observed on tunnels after earthquakes. The empirical curve produced by ALA (2001) in terms of PGA $\mathrm{gl}_{\mathrm{gl}}$ and relevant to minor damage - that is minor cracking of tunnel lining, minor rock falls, spalling of shotcrete or other supporting materials - is superimposed on the plot in Fig. 10(b). The comparison is satisfactory.

Moreover, superimposed on Fig. 10(d), there are the curves computed by Corigliano (2007) as a function of the $\mathrm{PGV}_{\mathrm{gl}}$ for deep tunnels corresponding to no or slight damage (i.e. cracking of the concrete lining) and moderate damage (e.g. spalling of the concrete lining, liner steel exposed, cracking and crushing of the concrete lining, etc.). Consistently with the class of the deep tunnel for which it was obtained, the empirical curve associated with moderate damage is practically coincident with the numerical curve corresponding to deep tunnels in dense soil and very close to the ones corresponding to deep tunnels in loose soil. On the other hand, the empirical curve for slight damage is far from the numerical predictions, probably because the first is 
associated with no or negligible effects, whereas the others are associated with the damage being sustained.

The numerical fragility curves showed a satisfactory agreement with the empirical curves, although the latter were obtained

(a) by applying a different fragility model, for which the probability of failure is computed as the probability of exceeding the IM when the DS was observed

(b) by collecting damage data felt by different types of tunnel lining embedded in heterogeneous soil types.

\section{CONCLUSIONS}

This paper investigates the dynamic soil-tunnel interaction mechanisms under different earthquakes with amplitudes expressed in terms of PGA, ranging from $0 \cdot 05 \mathrm{~g}$ to $0 \cdot 515 \mathrm{~g}$. Soil stiffness and tunnel depth were also varied to investigate their effects.

The presence of the tunnel was found to attenuate the surface motion since the high-frequency content of seismic waves is filtered by the void. This effect is only relevant under weak motions and tends to decrease when strong motions mobilise the soil non-linearity.

Earthquake-induced variations of internal forces were recognised in the lining, with greater changes in the deep tunnel, where stress levels are higher. Since critical reductions of the axial forces occurred in the shallow tunnels, the safety of the tunnel was assessed during the whole duration of the seismic excitation, taking into account the dependence of the bending resistance on the axial force.

The results were expressed in terms of fragility curves, considering different seismic IMs. The best correlation with the seismic performance of tunnels was obtained by the PGV recorded at the surface in free-field conditions, owing to its direct correlation with shear strains. Even though its effectiveness is shown, such a parameter is affected by the local site response, which is unknown immediately after an earthquake. For this reason, the same curves were expressed as a function of the input motion parameters to facilitate their application in real-time analyses in the post-earthquake management of infrastructures (Fabozzi et al., 2018; Fabozzi et al., 2019). The comparison revealed that tunnels in loose sand are less vulnerable to severe input motions, thanks to the seismic attenuation of soil non-linearity.

Furthermore, the satisfactory comparison with the empirical fragility curves confirms the hypothesis that the variation of axial force plays an important role in the seismic performance of tunnels.

Overall, the suitable evaluation of the seismic vulnerability of strategic infrastructure like tunnels has undoubtedly an important impact on the socio-economic resilience of a specific system or community. It should therefore be an integral part of the risk identification and management of linear infrastructure. The current availability of fragility curves for tunnels is very limited. This has a negative impact on the inclusion of these structures in streamlined risk management tools using geographical information systems or loss-driven earthquake early warning and rapid response systems (Fabozzi et al., 2018).

It seems worth noticing that one of the most recent developments of performance-based earthquake engineering is the expression of the annual rate of failure, obtained from the combined calculation of the fragility of the system under investigation and the seismic hazard on site. In its most refined form, the approach is intended to include in the definition of seismic risk site-specific effects that are neglected by the traditional uncoupled formulation of hazard and fragility. This approach is becoming increasingly common for assessing the seismic risk on structures (Jalayer \& Cornell, 2009; Iervolino et al., 2017), while a few pioneering applications have been developed by geotechnical researchers (Kramer, 2008, 2014). In this vein, fragility curves like those presented in the present work, generated from full dynamic analyses, improve the reliability of risk assessment, as they directly take into account site amplifications and soilstructure interaction.

\section{ACKNOWLEDGEMENTS}

The research was developed in the frame of the project 'Shaking tunnel vision' funded by the Engineering and Physical Sciences Research Council and 'Extended shaking tunnel vision' funded by the Global Challenge Research Funding - Higher Education Funding Council for England.

\section{NOTATION}

$a_{\mathrm{cc}, \max }$ maximum acceleration

$C / D$ tunnel cover/tunnel diameter

$D$ damping ratio

$D_{0} \quad$ minimum damping ratio

$D_{\mathrm{r}}$ relative density

$\mathrm{DCR}_{\mathrm{LS}}$ demand to capacity ratio, limit state

E Young's modulus

$e_{\max }$ maximum void ratio

$e_{\min }$ minimum void ratio

$F$ flexibility ratio

$f$ frequency

$f_{\max }$ maximum frequency

$f_{\min }$ minimum frequency

$G_{0} \quad$ initial shear modulus

$G_{\text {soil,mob }}$ mobilised shear modulus

$G / G_{0}$ normalised shear modulus

$H_{\text {axis }}$ depth of the tunnel axis

$K_{0} \quad$ bulk modulus

$\log \eta_{\mathrm{DCR}_{\mathrm{LS}} \mid \mathrm{IM}}$ mean value of the lognormal distribution of the demand-to-capacity ratio

$M_{\mathrm{W}}$ moment magnitude

$M_{\text {res }}$ residual bending moment

$m$ coefficient of the sigmoid function

$N$ axial force

$n$ coefficient of the sigmoid function

$n_{\mathrm{c}}$ homogenisation coefficient

$\mathrm{PGA}_{\mathrm{gl}}$ peak ground acceleration at ground level

$\mathrm{PGA}_{\mathrm{im}}$ peak ground acceleration of the input motion

PGV peak ground velocity

$\mathrm{PGV}_{\mathrm{gl}}$ peak ground velocity at ground level

$\mathrm{PGV}_{\mathrm{im}}$ peak ground velocity of the input motion

$p$ coefficient of the sigmoid function

$R_{\text {ck }} \quad$ concrete resistance

$R_{\text {epi }}$ epicentral distance

$r$ tunnel radius

$T_{\mathrm{P}}$ predominant period

$V_{\mathrm{S}}$ shear wave velocity

$\gamma$ shear strain

$\gamma_{\text {soil }}$ soil unit weight

$\Delta$ mesh size

$\Delta M \quad$ cyclic change of bending moment

$\lambda_{\max }$ maximum wavelength

$v$ Poisson's ratio

$\rho$ soil density

$\sigma_{\mathrm{cy}}$ concrete uniaxial compression strength

$\sigma_{\mathrm{DCR}_{\mathrm{LS}} \mid \mathrm{IM}}$ standard deviation of the lognormal distribution of the demand-to-capacity ratio

$\sigma_{\mathrm{rl}} \quad$ stress acting in the lower steel reinforcements

$\sigma_{\text {ru }}$ stress acting in the upper steel reinforcements

$\sigma_{\mathrm{t}}$ concrete uniaxial tensile strength

$\tau_{\text {eq }}$ equivalent shear stress

$\phi$ friction angle

$\chi \quad$ vector of parameters 


\section{REFERENCES}

Abate, G., Massimino, M. R. \& Maugeri, M. (2015). Numerical modelling of centrifuge tests on tunnel-soil systems. Bull. Earthquake Engng 13, No. 7, 1927-1951.

ALA (American Lifelines Alliance) (2001). Seismic fragility formulation for water systems, part 1. Reston, VA, USA: American Society of Civil Engineers (ASCE)-Federal Emergency Management Agency (FEMA).

Amorosi, A., Boldini, D. \& Falcone, G. (2014). Numerical prediction of tunnel performance during centrifuge dynamic tests. Acta Geotech. 9, No. 4, 581-596.

Andreotti, G. \& Lai, C. (2014). Seismic vulnerability of deep tunnels: numerical modelling for a fully nonlinear dynamic analysis. In 2nd European conference on earthquake engineering and seismology 2014 (2nd ECEES), vol. 6, pp. 4786-4798. Red Hook, NY, USA: Curran Associates, Inc.

Argyroudis, A. \& Pitilakis, K. (2012). Seismic fragility curves of shallow tunnels in alluvional deposits. Soil Dyn. Earthq. Engng 35, 1-12.

Argyroudis, S., Tsinidis, G., Gatti, F. \& Pitilakis, K. (2017). Effects of SSI and lining corrosion on the seismic vulnerability of shallow circular tunnels. Soil Dyn. Earthq. Engng 98, 244-256.

Bilotta, E. (2018). Modelling tunnel behaviour under seismic actions: an integrated approach. In Physical modelling in geotechnics (ICPMG 2018) (eds A. McNamara, S. Divall, R. Goodey, N. Taylor, S. Stallebrass and J. Panchal), vol. 1, pp. 3-20. Abingdon, UK: Taylor \& Francis Group.

Bilotta, E., Madabhushi, S. P. G. \& Silvestri, F. (2014a). Editorial: round robin tunnel test (RRTT). Acta Geotech. 9, No. 4, $561-562$.

Bilotta, E., Lanzano, G., Madabhushi, S. P. G. \& Silvestri, F. (2014b). A numerical round robin on tunnels under seismic actions. Acta Geotech. 9, No. 4, 563-579.

Broere, W. (2016). Urban underground space: solving the problems of today's cities. Tunn. Undergr. Sp. Technol. 55, No. May 2016, 245-248.

BSI (2004). BS EN 1992-1-1 (English): Eurocode 2: design of concrete structures - part 1-1: general rules and rules for buildings (Authority: The European Union per Regulation 305/2011, Directive 98/34/EC, Directive 2004/18/EC). London, UK: BSI.

Cilingir, U. \& Madabhushi, S. P. G., (2011a). A model study on the effects of input motion on the seismic behaviour of tunnels. Soil Dyn. Earthq. Engng 31, No. 3, 452-462.

Cilingir, U. \& Madabhushi, S. P. G. (2011b). Effect of depth on seismic response of circular tunnels. Can. Geotech. J. 48, No. 1, $117-127$.

Conti, R., Viggiani, G. M. B. \& Perugini, F. (2014). Numerical modelling of centrifuge dynamic tests of circular tunnels in dry sand. Acta Geotech. 9, No. 4, 597-612.

Corigliano, M. (2007). Seismic response of deep tunnels in near-fault conditions. $\mathrm{PhD}$ thesis, Politecnico di Torino, Turin, Italy.

Cubrinovski, M. \& Ishihara, K. (2002). Maximum and minimum void ratio characteristics of sands. Soils Found. 42, No. 6, 65-78.

de Silva, F. \& Scotto di Santolo, A. (2018). Probabilistic performance-based approaches to the static and seismic assessment of rock cavities. Int. J. Rock Mech. Min. Sci. 112, No. 12, 354-368.

de Silva, F., Pitilakis, D., Ceroni, F., Sica, S. \& Silvestri, F. (2018). Experimental and numerical dynamic identification of a historic masonry tower accounting for different types of interaction. Soil Dyn. Earthq. Engng 109, No. 6, 235-250.

Dobry, R., Oweis, I. \& Urzua, A. (1976). Simplified procedure for estimating the fundamental period of a soil profile. Bull. Seismol. Soc. Am. 66, No. 4, 1293-1321.

Dowding, C. H. \& Rozen, A., (1978). Damage to rock tunnels from earthquake shaking, Am. Soc. Civ. Engrs, J. Geotech. Engng Div. 104, No. 2, 175-191.

Fabozzi, S. (2017). Behaviour of segmental tunnel lining under static and dynamic loads. $\mathrm{PhD}$ dissertation, University of Naples Federico II, Naples, Italy.

Fabozzi, S. \& Bilotta, E. (2016). Behaviour of a segmental tunnel lining under seismic actions. VI CNRIG Italian Conference of Researchers in Geotechnical Engineering, Bologna, Italy, September 2016. Procedia Engng 158, 230-235.
Fabozzi, S., Bilotta, E. \& Lanzano, G. (2017). A numerical study on seismic vulnerability of tunnel linings. Proceedings of $3 \mathrm{rd}$ international conference on performance-based design in earthquake geotechnical engineering, Vancouver, Canada.

Fabozzi, S., Bilotta, E., Picozzi, M. \& Zollo, A. (2018). Feasibility study of a loss-driven earthquake early warning and rapid response system for tunnels of Italian high-speed railway network. Soil Dyn. Earthq. Engng 112, 232-242.

Fabozzi, S., Bilotta, E., Picozzi, M. \& Zollo, A. (2019). Feasibility study of earthquake early warning systems: the case of the Italian highspeed railway network. In Earthquake geotechnical engineering for protection and development of environment and constructions (eds F. Silvestri and N. Moraci), pp 2291-2298. Leiden, the Netherlands: CRC Press/Balkema.

Gomes, R. C. (2014). Numerical simulation of the seismic response of tunnels in sand with an elastoplastic model. Acta Geotech. 9, No. 4, 613-629.

Hardin, B. O. (1978). The nature of stress-strain behaviour for soils. In Earthquake engineering and soil dynamics, pp. 3-90. New York, NY, USA: American Society of Civil Engineers.

Hleibieh, J., Wegener, D. \& Herle, I. (2014). Numerical simulation of a tunnel surrounded by sand under earthquake using a hypoplastic model. Acta Geotech. 9, No. 4, 631-640.

Hoeg, K. (1968). Stresses against underground structural cylinders. J. Soil Mech. Found. Div. 94, No. 4, 833-858.

Iervolino, I., Spillatura, A. \& Buzzurro, P. (2017). RINTC PROJECT: assessing the (implicit) seismic risk of codeconforming structures in Italy. In COMPDYN 2017: 6th ECCOMAS thematic conference on computational methods in structural dynamics and earthquake engineering methods in structural dynamics and earthquake engineering (eds M. Papadrakakis and M. Fragiadakis), pp. 1545-1557. Brussels, Belgium: ECCOMAS Proceedia, https://doi.org/10. 7712/120117.5512.17282.

Itasca (Itasca Consulting Group) (2011). FLAC 7.0 - fast Lagrangian analysis of Continua - user's guide. Minneapolis, MN, USA: Itasca Consulting Group.

Jalayer, F. \& Cornell, C. A. (2009). Alternative nonlinear demand estimation methods for probability-based seismic assessments. Earthq. Engng Struct. Dyn. 38, No. 8, 951-972.

Jalayer, F., De Risi, R. \& Manfredi, G. (2015). Bayesian cloud analysis: efficient structural fragility assessment using linear regression. Bull. Earthquake Engng 13, No. 4, 1183-1203.

Jalayer, F., Ebrahimian, H., Miano, A., Manfredi, G. \& Sezen, H. (2017). Analytical fragility assessment using unscaled ground motion records. Earthq. Engng Struct. Dyn. 46, No. $15,2639-2663$.

Kampas, G., Knappett, J. A., Brown, M. J., Anastasopoulos, I., Nikitas, N. \& Fuentes, R. (2019). The effect of tunnel lining modelling approaches on the seismic response of sprayed concrete tunnels in coarse-grained soils. Soil Dyn. Earthq. Engng 117, 122-137.

Kiani, M., Ghalandarzadeh, A., Akhlaghi, T. \& Ahmadi, M. (2016). Experimental evaluation of vulnerability for urban segmental tunnels subjected to normal surface faulting. Soil Dyn. Earthq. Engng 89, 28-37.

Kontoe, S., Avgerinos, V. \& Potts, D. M. (2014). Numerical validation of analytical solutions and their use for equivalentlinear seismic analysis of circular tunnels. Soil Dyn. Earthq. Engng 66, 206-219.

Kramer, S. L. (2008). Performance-based earthquake engineering: opportunities and implications for geotechnical engineering practice. In Geotechnical earthquake engineering and soil dynamics IV (eds D. Zeng, M. T. Manzari and D. R. Hiltunen), Geotechnical Special Publication 181, CD-ROM. Reston, VA, USA: American Society of Civil Engineers.

Kramer, S. L. (2014). Performance-based design methodologies for geotechnical earthquake engineering. Bull. Earthq. Engng 12, No. 3, 1049-1070.

Kuhlemeyer, R. L. \& Lysmer, J. (1973). Finite element method accuracy for wave propagation problems. J. Soil Mech. Found. Div. 99, No. 5, 421-427.

Lanzano, G., Bilotta, E., Russo, G., Silvestri, F. \& Madabhushi, S. P. G. (2012). Centrifuge modelling of seismic loading on tunnels in sand. Geotech. Test J. 35, No. 6, 854-869. 
Masing, G. (1926). Eigenspannungen und verfertigung bim messing. In Proceeding of 2nd international congress on applied mechanics, pp. 322-355. Zürich, Switzerland: Orell Füssli (in German).

NIBS (National Institute of Building Sciences) (2004). HAZUS-MH: user manual and technical manuals, report. Washington, DC, USA: Federal Emergency Management Agency.

Ochmański, M., Delli Carpini, M., Salvatore, E., Bilotta, E. \& Modoni, G. (2019). Numerical simulation of centrifuge seismic tests on tunnel in sandy soil. In Earthquake geotechnical engineering for protection and development of environment and constructions (eds F. Silvestri and N. Moraci), pp. 4162-4170. Leiden, the Netherlands: CRC Press/Balkema.

O'Rourke, M. J. \& Liu, X. (1999). Response of buried pipelines subject to earthquake effects. Buffalo, NY, USA: Multidisciplinary Center for Earthquake Engineering Research.

Papadimitriou, A. G. \& Bouckovalas, G. D. (2002). Plasticity model for sand under small and large cyclic strains: a multiaxial formulation. Soil Dyn. Earthq. Engng 22, No. 3, 191-204.

Pelecanos, L., Kontoe, S. \& Zdravkovic, L. (2015). A case study on the seismic performance of earth dams. Géotechnique $\mathbf{6 5}$, No. 11, 923-935, https://doi.org/10.1680/jgeot.SIP.15.P.009.

Pitilakis, K., Crowley, H. \& Kaynia, A. (eds) (2014). SYNER-G: typology definition and fragility functions for physical elements at seismic risk, buildings, lifelines, transportation networks and critical facilities, p. 27. Dordrecht, the Netherlands: Springer.

Power, M. S., Rosidi, D. \& Kaneshiro, J. Y. (1998). Seismic vulnerability of tunnels and underground structures revisited. In North American tunneling '98 (ed. L. Ozdemir), pp. 243-250. Rotterdam, the Netherlands: Balkema.
Rayleigh, L. (1945). Theory of sound. New York, NY, USA: Dover Publications.

Salmon, M., Wang, J., Jones, D. \& Wu, C. (2003). Fragility formulation for the BART system. In Advancing mitigation technologies and disaster response for lifeline systems (ed. J. E. Beaver), pp. 183-192. Reston, VA, USA: American Society of Civil Engineers.

Seed, H. B. \& Idriss, I. M. (1970). Soil moduli and damping factors for dynamic response analyses, Report no. EERC70-10. Berkeley, CA, USA: Earthquake Engineering Research Center, University of California.

Sharma, S. \& Judd, W. R. (1991). Underground opening damage from earthquakes. Engng Geol. 30, No. 3-4, 263-276.

Silvestri, F. \& Lanzo, G. (1999). Risposta sismica locale: teoria ed esperienze. Benevento, Italy: Hevelius (in Italian).

Tropeano, G., Soccodato, F. M. \& Silvestri, F. (2018). Re-evaluation of code-specified stratigraphic amplification factors based on Italian experimental records and numerical seismic response analyses. Soil Dyn. Earthq. Engng 110, 262-275.

Tsinidis, G., Pitilakis, K. \& Trikalioti, A. D. (2014). Numerical simulation of round robin numerical test on tunnels using a simplified kinematic hardening model. Acta Geotech. 9, No. 4, 641-659.

Tsinidis, G., Pitilakis, K. \& Anagnostopoulos, C. (2016). Circular tunnels in sand: dynamic response and efficiency of seismic analysis methods at extreme lining flexibilities. Bull. Earthquake Engng 14, No. 10, 2903-2929.

Wang, J. N. (1993). Seismic design of tunnels: a simple state of the art design approach. New York, NY, USA: Parsons Brinckerhoff, Inc. 